Accepted for publication in the AJ

\title{
Spectroscopy of Bright QUEST RR Lyrae Stars: Velocity Substructures toward Virgo
}

\author{
A. Katherina Vivas ${ }^{1}$, Yara L. Jaffé ${ }^{1,2}$, Robert Zinn ${ }^{3}$, Rebeccah Winnick ${ }^{3}$, Sonia Duffau ${ }^{4}$, \\ Cecilia Mateu ${ }^{1}$
}

\begin{abstract}
Using a sample of 43 bright $(V<16.1$, distance $<13 \mathrm{kpc}$ ) RR Lyrae stars (RRLS) from the QUEST survey with spectroscopic radial velocities and metallicities, we find that several separate halo substructures contribute to the Virgo overdensity (VOD). While there is little evidence for halo substructure in the spatial distribution of these stars, their distribution in radial velocity reveals two moving groups. These results are reinforced when the sample is combined with a sample of blue horizontal branch stars that were identified in the SDSS, and the combined sample provides evidence for one additional moving group. These groups correspond to peaks in the radial velocity distribution of a sample of $\mathrm{F}$ type main-sequence stars that was recently observed in the same directon by SEGUE, although in one case the RRLS and F star groups may not lie at the same distance. One of the new substructures has a very narrow range in metallicity, which is more consistent with it being the debris from a destroyed globular cluster than from a dwarf galaxy. A small concentration of stars have radial velocities that are similar to the Virgo Stellar Stream (VSS) that was identified previously in a fainter sample of RRLS. Our results suggest that this feature extends to distances as short as $\sim 12 \mathrm{kpc}$ from its previous detection at $\sim 19$ kpc. None of the new groups and only one star in the sample have velocities that are consistent with membership in the leading tidal stream from the Sagittarius Dwarf Spheroidal Galaxy, which some authors have suggested is the origin of the VOD.
\end{abstract}

\footnotetext{
${ }^{1}$ Centro de Investigaciones de Astronomía (CIDA), Apartado Postal 264, Mérida 5101-A, Venezuela

${ }^{2}$ School of Physics \& Astronomy, University of Nottingham, University Park, Nottingham NG7 2RD, UK

${ }^{3}$ Department of Astronomy, Yale University, P.O. Box 208101, New Haven, CT 06520-8101

${ }^{4}$ Departamento de Astronomía, Universidad de Chile, Casilla 36-D, Santiago, Chile
} 
Subject headings: stars: variables: other - Galaxy: halo - Galaxy: kinematics - Galaxy: structure

\section{INTRODUCTION}

Over the past several decades, a large number of investigations of the stars and the globular clusters in the Galactic halo have concluded that the halo formed chaotically and over a long period of time through the accretion and disruption of dwarf galaxies (e.g., Searle \& Zinn 1978, Norris 1986; Carney et al. 1990; Majewski 1992; Mackey \& Gilmore 2004; see also Freeman \& Bland-Hawthorn 2002 for a review). There is evidence, however, that the inner and outer parts of the halo have sufficiently different properties that at least two mechanisms are required to explain the halo (e.g. Hartwick 1987; Zinn 1993; Majewski 1993; Chiba \& Beers 2000; Kinman et al. 2007; Miceli et al. 2007; Lee et al. 2007; Carollo et al. 2007). Accretion appears to have dominated the formation of the outer halo, but the flattening and prograde rotation of the inner halo and the old ages of the globular clusters in the inner halo suggest that its formation involved the dissipative merging of gas clouds at an early epoch (see Carollo et al. 2007, and references therein). While this dual halo picture is supported by substantial evidence, it continues to be important to see what fraction of the halo can be explained by, for example, an accretion-only scenario.

The most direct evidence for accretion comes from the detection of halo substructures consisting of co-moving streams of stars. The best documented of these are the streams originating in the Sagittarius (Sgr) dwarf spheroidal (dSph) galaxy, which cross much of the sky (Yanny et al. 2000; Ivezić et al. 2000; Ibata et al. 2001b; Vivas et al. 2001, 2005; Newberg et al. 2002; Majewski et al. 2003, among others). Several other substructures have been recently detected in the halo of the Milky Way (Newberg et al. 2002; Ibata et al. 2003; Majewski et al. 2003; Yanny et al. 2003; Rocha-Pinto et al. 2004; Vivas \& Zinn 2006; Belokurov et al. 2006, among others) and in the halo of M31 (Ibata et al. 2001a; Ferguson et al. 2002; Morrison et al. 2003; Zucker et al. 2004; Kalirai et al. 2006; Fardal et al. 2007). These streams provide strong evidence in favor of the hierarchical model of the formation of galaxies, in which large galaxies such as the Milky Way formed through multiple mergers with smaller building blocks. Simulations of the hierarchical picture predict that the Milky Way has experienced a large number of minor mergers (see for example Bullock \& Johnston 2005) and show that the ones that occurred during the past few Gyrs. may be recognizable today as stellar streams. While these simulations appear to be consistent with the current observations of the outer halo (Bell et al. 2007), the search for more streams and better documentation of the known ones remains important. 
We report here an investigation of the halo substructure in the direction of Virgo. The first sign of an overdensity of halo stars in this direction came from the QUEST RR Lyrae star (RRLS) survey (Vivas et al. 2001), which revealed a small overdensity near $13 \mathrm{hr}$ right ascension $(\alpha)$, which was the west boundary of the survey as it existed then. As the survey was extended, it became clear that this feature is considerably larger and is centered near $\alpha \sim 12.4 \mathrm{hr}$ (Vivas 2002; Vivas \& Zinn 2003). In the jargon of the QUEST survey it became known as the "12.4 hr clump" (declination $(\delta)$ was not specified because of the narrow range of the survey around $\delta=-1^{\circ}$ ). The RRLS in the clump cover a range of distances from the Sun $(D)$ with the greatest concentration at $D \sim 19 \mathrm{kpc}$ (Vivas \& Zinn 2006). Using photometry from the SDSS, Newberg et al. (2002) independently discovered an overdensity in $\mathrm{F}$ type main-sequence stars in approximately the same direction and at a similar distance, which they called S297+63-20.5. They suggested that it may be related to the small group of RRLS identified earlier by Vivas et al. (2001), and several authors have subsequently suggested that the $12.4 \mathrm{hr}$ clump of RRLS and S297+63-20.5 are the same substructure or at least related. Also using the SDSS photometric catalogue, Juric et al $(2005,2008)$ later identified a very large overdensity of main sequence stars toward Virgo (the Virgo Overdensity (VOD) ). On the sky, the VOD encompasses both the $12.4 \mathrm{hr}$ clump and S297+63-20.5. But, according to Juric et al. (2008), the VOD is spread along the line of sight from $\sim 6$ to $\geq 20 \mathrm{kpc}$, which is a larger range than either the $12.4 \mathrm{~h}$ clump or S297+63-20.5. The spectroscopy of RRLS in the $12.4 \mathrm{hr}$ clump by Duffau et al. (2006) provided the first evidence of a moving group in the Virgo direction, which they called the Virgo Stellar Stream (VSS). The recent spectroscopy of F type main-sequence stars in S297+63-20.5 by Newberg et al. (2007) revealed a rich structure in velocity space, but with a major peak that nearly coincides with the velocity of the VSS. They tentatively conclude that S297+63-20.5, the VSS, and the VOD are likely associated, if not the same. On the other hand, several authors (Majewski et al. 2003; Martínez-Delgado et al. 2004, 2007) have argued that the Virgo substructures are parts of Sgr streams, while others (Newberg et al. 2002, 2007; Jurić et al. 2008; Duffau et al. 2006) have rejected this hypothesis and suggested instead that they are due to a separate merger event involving a dwarf galaxy.

We are carrying out a program to observe spectroscopically a large number of QUEST RRLS in order to measure their radial velocities and metallicities. RRLS are arguably the halo tracer that can be most precisely located in space, and with the addition of radial velocities of even modest precision $\left( \pm 20 \mathrm{~km} \mathrm{~s}^{-1}\right)$, they become powerful probes of phase space. This wonderful property of RRLS comes with a price, for they are expensive observationally. Complete light curves are necessary to obtain both mean magnitudes (hence, precise distances) and ephemerides. The later are necessary to isolate the motion of the star in the sky from the pulsational velocity of the star by means of the fitting of a radial velocity curve. In 
this paper, we present spectroscopic observations of bright RRLS ( $V_{0}<16.1$, equivalent to D $\lesssim 12.5 \mathrm{kpc}$ ) in the part of the QUEST survey that overlaps with the VOD. After examining these data for halo substructure, we discuss the relationships between our results and the previous detections of substructures in Virgo and also consider the possibility that they are related to streams from the Sgr dSph galaxy. The RRLS surveys by Keller et al. (2007) and

by Wilhelm et al. (2007) provide important data on parts of the VOD that are not covered by the QUEST survey.

\section{THE DATA}

\subsection{The Sample of RR Lyrae Stars}

According to Jurić et al. (2008), the VOD occupies $\sim 1000$ sq. degrees of the sky, and its rough limits are shown in Figure1. Notice that the SDSS does not extend south of $b \sim 58^{\circ}$ at $l \sim 300^{\circ}$ (see Figure 6 in Jurić et al. 2008). Thus, it is possible that the VOD extends farther in that direction. The region surveyed by QUEST is a 2.3 -wide band centered at $\delta=-1^{\circ}$. It passes over part of the VOD, as shown in the Figure 1, The dot-dashed line in Figure 1 shows the southern limit of a second QUEST catalog (Vivas et al. in preparation) which is centered at $\delta=-3^{\circ}$. Eight stars from this second region are included in our sample.

The region in common between the VOD and the QUEST survey is roughly limited by galactic longitude in the range $260^{\circ} \lesssim l \lesssim 340^{\circ}$. At the declination of the QUEST survey, those limits are equivalent to $169^{\circ} .5<\alpha<212^{\circ} .3$. We have therefore selected for spectroscopy all RRLS in the QUEST catalog (Vivas et al. 2004) within these limits in $\alpha$, and with magnitude $V \leq 16.1$. The constraint in magnitude was imposed by the limitations of our major instrument, the SMARTS $1.5 \mathrm{~m}$ telescope (see $\S 2.2$ ). The magnitudes of this sample correspond to a range of distance from the Sun of $\sim 4$ to $12.5 \mathrm{kpc}$ (using $M_{V}=0.55$, following Vivas \& Zinn 2006), which partly overlaps the range estimated for the $\operatorname{VOD}(\sim 6-20 \mathrm{kpc}$, Jurić et al. 2008).

Figure 2 shows the extinction corrected magnitude $V_{0}$ (equivalent to distance) of part of the QUEST RRLS in the Northern Galactic Hemisphere, as a function of $\alpha$. The box encloses the sample of 44 stars studied in this work. Within the box, all the stars in the first QUEST catalog were observed spectroscopically, but only 8 RRLS (out of 38) from the second QUEST catalog were observed. For reference, we have indicated with solid symbols the location of the RRLS that probably belong to the VSS (Duffau et al. 2006). The stars in our sample are located in the same area of the sky as the VSS, but are closer to the Sun. Most of them (the ones with $D>6 \mathrm{kpc}$ ) are located within the same region of the sky and 
distance range as the VOD. Notice also in Figure 2 that part of the tidal stream of the Sgr dSph galaxy lies in the background, at $\sim 50 \mathrm{kpc}$.

\subsection{Spectroscopy}

We obtained a total of 88 spectra of the 44 RRLS with 3 different telescopes between 2001 and 2007. The instrumental setups of the different observing runs are summarized in Table 1, and the individual observations are detailed in Table 2. Most of the spectra were obtained with the SMARTS $1.5 \mathrm{~m}$ telescope at Cerro Tololo Interamerican Observatory, Chile (denoted as "SMARTS" in Table 2). We also used observations obtained with the $1.5 \mathrm{~m}$ telescope in La Silla, Chile (denoted as "ESO") and with the Hydra multifiber spectrograph at WIYN in Kitt Peak National Observatory, USA. Most of the spectra were taken at blue wavelengths, covering the Balmer lines (beginning in $\mathrm{H} \beta$ ) and the $\mathrm{Ca}$ II $\mathrm{H}$ and $\mathrm{K}$ lines. However, we took advantage of available time from other projects at the WIYN telescope and obtained spectra using a setup which covered the red part of the spectra, including the Ca triplet lines. The WIYN observations are denoted in Table 2 as "WIYN-B" and "WIYN-R" for blue and red spectra respectively.

For each target star, we tried to obtain at least two spectra taken at different phases during the pulsation cycle. Thirty five out of the 44 RRLS have two or more spectra available. Table 2 contains ID (same QUEST ID as in Vivas et al. 2004), Julian date, exposure time, telescope, phase of observation (see $\S 2.3$ ), heliocentric radial velocity with its error (see $\S$ 2.5), and metallicity (see $\S 2.4$ ). The 8 stars from the unpublished second part of the QUEST catalog have ID numbers higher than 700.

The spectrum of star \#254 indicates that is too cool to be a RRc star. This is not surprising because a small fraction of the QUEST RR $c$ stars are expected to be misidentifications of other types of variable stars, mainly variable blue stragglers and W UMa eclipsing binaries (see Vivas et al. 2004). Star \# 254 was excluded from any further analysis.

To avoid excessive broadening in the spectral features due to the changing velocity of the star during the pulsational cycle, exposure times were kept short, usually under 30 minutes. A few stars have exposure times as long as 60 minutes, which is equivalent to $\sim 10 \%$ of the pulsation cycle of a typical RRLS. In all observing runs, we observed several stars from the list of Layden (1994) which are both radial velocity and pseudo-equivalent width standards. 


\subsection{Improving the Ephemerides}

Since good ephemerides are essential for fitting the radial velocity curves, it is important to improve them as much as possible. The QUEST survey has a large number of epochs (an average 32 for our targets), which should yield precise ephemerides. In Vivas et al. (2004), the time of maximum light $\left(\mathrm{HJD}_{0}\right)$ for the $\mathrm{RR} c$ stars was set by the observation with the brightest magnitude, which is not the best procedure because it can be skewed by photometric error and by poor sampling near maximum light. In addition, for some $\mathrm{RR} c$ stars, it was necessary to list two possible periods because both produced reasonable light curve shapes to the eye.

To obtain better light curve parameters, we have fitted a light curve template to each $\mathrm{RR} c$ star in our sample. A sine curve produced a poor fit because a typical RR $c$ stars has a phase difference between maximum and minimum light of $\sim 0.4$. Consequently, we constructed a light curve template based on 9 of the best observed QUEST RR $c$ stars, which did not suffer from period aliases or from a poorly measured $\mathrm{HJD}_{0}$. This template was then fitted using $\chi^{2}$ minimization, while varying the amplitude, phase at maximum light, magnitude of maximum light, and period around the values listed in the QUEST catalogue. We also explored the parameters around the $1 / P \pm 1$ alias periods.

The ephemerides for the RR $a b$ stars in the QUEST catalogue were more secure because they were already determined from light curve fitting and because period aliases were less of a problem for them. However, the period was not allowed to vary in the fitting. This time it was allowed to vary in the fitting, but the resulting light curves are not significantly different from the old ones. Only in 5 cases were the changes in period equal to or slightly larger than $2 \times 10^{-5}$ days. The ephemerides that yielded the best fits to the light curves for the RR $c$ and RRab stars have been adopted here and are listed in Table 3 ,

\subsection{Measurements of $[\mathrm{Fe} / \mathrm{H}]$}

The metallicities of the RRLS have been measured from the spectrograms following the methodology and calibration of Lavden (1994), which is based on Freeman \& Rodgers' (1975)

modification of the Preston (1959) $\Delta \mathrm{S}$ method. The method, which involves plotting the pseudo-equivalent width of the Ca II K line $(\mathrm{W}(\mathrm{K}))$, corrected for interstellar absorption, against the mean pseudo-equivalent widths of the $\beta, \gamma$, and $\delta$ Balmer lines $(\mathrm{W}(\mathrm{H}))$, is described in detail in Vivas et al. (2005).

In general, the type c RRLS are less numerous than the type ab in old stellar populations, e.g. globular clusters, and because they are harder to detect than type ab, their number in 
RRLS surveys of the field suffer more from incompleteness. Layden (1994) did not produce a $[\mathrm{Fe} / \mathrm{H}]$ calibration for type c RRLS, which were ignored in his study of the metallicities and kinematics of field RRLS. There is a sufficient number of type $\mathrm{c}$ stars in our sample that it is important to measure $[\mathrm{Fe} / \mathrm{H}]$ from our spectrograms.

The average type $\mathrm{c}$ variable is $\sim 600 \mathrm{~K}$ hotter and has a $\sim 0.15$ dex larger surface gravity than the average type ab variable, although the types overlap in both quantities, particularly when the type ab variables are hottest during their pulsations. The Layden calibration may therefore require no modification when measuring type c RRLS. This is supported by Kemper's (1982) demonstration that the $\Delta \mathrm{S}$ method, which was devised for type ab variables, can be applied to type c, and more directly by Gratton et al. (2004), who found that both types of variables in same globular cluster define a tight sequence in a plot of $\mathrm{K}$ line strength against hydrogen line strength with some intermingling of the types along the sequence. However, Gratton et al. (2004) defined their K line and H line strengths differently than did Layden (1994).

To see if Layden's calibration can be applied to the type c, we observed both type c and type ab variables in the globular cluster M3 with the Hydra multiobject spectrograph on the WIYN telescope. While the fiber setup targeted a large number of variables of both types in this variable-rich cluster, the combination of partly cloudy weather and variable fiber throughputs limited the number of usable spectra to 8 type c stars and 10 type ab. Analyses of high dispersion spectrograms of red giants (Sneden et al. 2004) and RRLS (Sandstrom et al. 2001) and the tightness of the red giant branch in the color-magnitude diagram of M3 indicate that there is very little if any variation in $[\mathrm{Fe} / \mathrm{H}]$ among the stars in this cluster. For our purposes, we assume that there is no real star to star scatter and investigate whether Layden's calibration yields the same $[\mathrm{Fe} / \mathrm{H}]$ when applied separately to the two types of RRLS.

Our measurements for the M3 RRLS in the Layden pseudo-equivalent width system are plotted in Figure 3, where different symbols are used to depict the type ab and type c stars. The mean $[\mathrm{Fe} / \mathrm{H}]$ obtained from all 10 of the type ab variables is -1.78 with a standard deviation of 0.27 . The large scatter is undoubtedly due to the low $\mathrm{S} / \mathrm{N}$ of some of the spectrograms. The 5 type ab RRLS (filled squares in Figure 3i) for which we have the highest $\mathrm{S} / \mathrm{N}$ spectrograms $(50>S / N>17$ at the CaII K line) yield $\langle[\mathrm{Fe} / \mathrm{H}]\rangle=-1.72$ with a more reasonable std. dev. of 0.14 . The 8 type $\mathrm{c}$ variables yield $\langle[\mathrm{Fe} / \mathrm{H}]\rangle=-1.66$ (std. dev. $=0.17$ ), and the 5 that have spectrograms with $41>S / N>17$ yield $\langle[\mathrm{Fe} / \mathrm{H}]\rangle=-1.63$ (std. dev. $=0.19)$. The high $\mathrm{S} / \mathrm{N}$ samples differ in $\langle[\mathrm{Fe} / \mathrm{H}]\rangle$ by $0.09 \pm 0.11$, which suggests that the offset, if any, between the type ab and c variables is small. Since these mean values agree well with Zinn \& West's (1984) result for M3 $([\mathrm{Fe} / \mathrm{H}]=-1.66$ with an internal precision of 
\pm 0.06 ), Layden's calibration appears to be equally applicable to type c and type ab RRLS.

The metallicities that we have derived from the spectrograms of our program stars are listed in the last column of Table 2, The majority of the spectrograms have $S / N>20$, and from the experiences with the M3 variables, we estimate that the precision obtained from one spectrogram is \pm 0.15 for the type ab variables and \pm 0.20 for the type $c$. The lower precision for the type c RRLS is consequence of the convergence of the constant $[\mathrm{Fe} / \mathrm{H}]$ lines at large $\mathrm{W}(\mathrm{H})$ (see Figure 3 ). A few spectrograms have $S / N \sim 15$, and the $[\mathrm{Fe} / \mathrm{H}]$ values derived from them are marked with colons in Table 2. Layden's calibration breaks down for type ab variables that are observed on the rising branch, where the strength of the $\mathrm{K}$ line is reduced by the relatively large effective gravity and shock waves may produce emission in the cores of the $\mathrm{H}$ lines that reduce their equivalent widths. We have discarded measurements $[\mathrm{Fe} / \mathrm{H}]$ that were made at phases where these effects are likely to be important. The surface gravity variations of the type $\mathrm{c}$ variables are milder, and the equivalent widths of the $\mathrm{H}$ lines are not affected by emission. None of their measurements have been discarded.

\subsection{Radial Velocities}

Radial velocities for all blue spectra were obtained by Fourier cross-correlation (IRAFs fxcor task) with radial velocity standard stars of $\mathrm{A}$ and $\mathrm{F}$ spectral type, in the spectral range 3800 - $5200 \AA$. The radial velocity standards were separated in two groups according to their effective temperature. Each target star was cross-correlated with 6 to 14 different standard spectra of the group with more similar effective temperature. For each spectrum, we obtained a mean radial velocity, $V_{r}$, by weighting the result from each standard by the cross-correlation error returned by fxcor. This error depends on both the $\mathrm{S} / \mathrm{N}$ of the spectra and the similarity between the target star and the standard.

In order to estimate the radial velocity errors produced by the different telescopespectrograph combinations, we cross-correlated each spectrum of the radial velocity standards with the spectra of the other standards, taking care to avoid cross-correlating two spectra of the same star. Reassuringly, the differences between the mean velocities from these correlations and the literature values were close to zero for all of the standards with each instrumental setup. We adopted the standard deviations of these differences as the minimum error in velocity that we can achieve with a particular setup for our target stars $(16,8$, and $12 \mathrm{~km} / \mathrm{s}$ for the SMARTS, WIYN blue, and ESO 1.5m spectra, respectively). Because these errors include the uncertainties in the zero-points of the wavelength calibrations, we believe that these values better reflect the true errors than the means of the cross-correlation errors from fxcor, which are smaller. 
Cross-correlation was not used to calculate the radial velocities of the red Hydra spectra because the subtraction of the strongest night-sky lines left residuals in some wavelength regions. Instead, Gaussian line profiles were fitted to the unblended Paschen lines of hydrogen and/or the two strongest lines of Ca II, which however at the resolution of the spectrograms are each blended with a Paschen line. The Ca II lines were only measured in spectra that were taken at the coolest phases of the type ab variables when the Paschen lines are much weaker than the Ca II lines. Before computing the radial velocities from the Ca II lines, we made slight adjustments to their wavelengths to take into account the blending. These were estimated from high $\mathrm{S} / \mathrm{N}$ spectrograms of bright stars of known velocity whose spectra resemble the type ab variables at minimum light. To test this method, we obtained with the SMARTS $1.5 \mathrm{~m}$ telescope red spectra of 6 bright type ab RRLS during cloudy weather. The velocities obtained from the measurements of the Ca II lines did not systematically deviated from the ones measured from the Paschen lines alone, and the systemic velocities (see below) obtained for these stars agreed with values in the literature. For the red Hydra spectra, the standard deviation of the mean of the velocities given by the individual lines ranged from 4 to $10 \mathrm{~km} \mathrm{~s}^{-1}$, which is consistent with the results obtained from similar spectra of bright stars of known velocity.

The best technique for obtaining the radial velocity of the center of mass (the systemic velocity, $V_{\gamma}$ ) of a type ab RRLS is to integrate the radial velocity curve given by weak metal lines over the whole pulsational cycle, and the results obtained for RRLS in globular clusters (e.g. Storm et al. 1992) are consistent with the radial velocities of the clusters to within the internal velocity dispersions of a few $\mathrm{km} \mathrm{s}^{-1}$. In our low resolution spectrograms, the weak lines produce poorly determined peaks in the cross-correlations, whereas well-defined ones are produced by the Balmer lines and other strong features. Furthermore, we could not obtain sufficiently large numbers of spectra to determine the velocity curve for each star. For the RRab, we adopted instead the method described by Layden (1994), which involves measuring the velocities at a few phases $(\phi)$ from low resolution spectrograms and then fitting them with the radial velocity curve of the ab variable X Arietis that Oke (1966) obtained from measurements of $H \gamma$. Observations made near maximum light $(\phi<0.1$ and $\phi>0.85)$ were not used in these fits because the radial velocity curve undergoes a large discontinuity in this region (see for example Smith 1995). The velocity curve that is determined from the $\mathrm{H}$ lines has a larger amplitude and differs in other ways from the one given by the metals lines (see Oke et al. 1962; Oke 1966). Nonetheless, Layden's (1994) comparisons of his measurements of $V_{\gamma}$ with literature values for the same stars indicates that this method produces results that are consistent with the errors of the measurements and the fits of the velocity curve. In the case of the template star X Ari, the systemic velocity is equal to the $\mathrm{H}$ line velocity at $\phi=0.5$, but there is some small star to star variation in this value. In our 
calculation of the errors in $V_{\gamma}$, we have included terms to account for this variation and for the variation in the amplitudes of the radial velocity curve (see Vivas et al. 2005). For RR $c$ stars, which have much smaller velocity amplitudes than the RRab, there are at most small offsets between the velocity curves given by the metal lines and the $\mathrm{H}$ lines. To measure $V_{\gamma}$, we fitted a template constructed by combining the radial velocity curves of $\mathrm{T}$ Sex and DH Peg (see Duffau et al. 2006).

Figure 4 shows the best fit for each star having two or more observations. These plots include all of the radial velocity measurements for a star, even if they were not used in the fits because they are close to $\phi=0$. In each case, these points lie within the expected range, which provides a check that our measurements do not suffer from large systematic errors. Notice also that six of the stars were observed with two different telescopes, and the consistency of the measurements, as seen in Figure 4, is also an indication that large systematic errors are not present.

In the fitting of the radial velocity curves, the data were weighted by their errors. The mean difference between the data points and the radial velocity curve, $\sigma_{\text {fit }}$, in most cases was of the same size, or smaller, than the typical errors in the individual measurements (see Table 3 ).

For the RR $c$, we calculated the error in the systemic velocity by combining the individual $\sigma_{r}$ in the usual manner for calculating the $\sigma$ of a weighted mean value. For both types of stars, if $\sigma_{\text {fit }}$ was larger than $\sigma_{\gamma}$ we adopted the former as our final error in the systemic velocity. The mean error in the systemic velocities of all, but one, RRLS range from 7 to $28 \mathrm{~km} \mathrm{~s}^{-1}$, with a mean value of $17 \mathrm{~km} \mathrm{~s}^{-1}$. One star, the type c \#174, had an unusually bad fit of the radial velocity curve and its final error is $42 \mathrm{~km} \mathrm{~s}^{-1}$. Finally, we calculated the radial velocity measured by an observer at the Sun who is at rest with the galactic center, $V_{g s r}$ by

$$
V_{g s r}=V_{\gamma}+10.0 \cos b \cos l+225.2 \cos b \sin l+7.2 \sin b
$$

Our results are presented in Table 3 , which lists: ID, coordinates $\alpha$ and $\delta$, the extinction corrected mean V magnitude, the type of RR Lyrae star, the period and Julian date at maximum light (see $\S 2.3$ ), distance from the Sun (D), number of spectra available and number of spectra actually used in the fitting of the radial velocity curve, the error in the fit of the radial velocity curve $\left(\sigma_{\text {fit }}\right)$, the systemic velocity $\left(V_{\gamma}\right)$ and its error $\left(\sigma_{\gamma}\right)$, and the velocity in the galactic rest frame, $\left(V_{g s r}\right)$. 


\section{DISTRIBUTION OF RADIAL VELOCITIES: SUBSTRUCTURES IN VELOCITY SPACE}

As noted above, the VOD encompasses the entire range in $\alpha$ and perhaps a larger range of distance than our sample of RRLS. The spatial distribution of our sample of RRLS (see the box in Figure 2) does not reveal any regions of exceptionally high density that are strong candidates for halo substructure. There is a roughly uniform band of stars that crosses the region at distances between 7 and $10 \mathrm{kpc}$ and also a region of relatively high density near $12 \mathrm{kpc}$ within $180^{\circ}<\alpha<195^{\circ}$. This second feature is the near side of the large overdensity in RRLS that we have referred to as the "12.4 hr clump" (Vivas 2002; Zinn et al. 2004; Vivas \& Zinn 2006), which at $19 \mathrm{kpc}$ contains the VSS (Duffau et al. 2006). Figure 12 in Vivas \& Zinn (2006) shows the location of the $12.4 \mathrm{hr}$ clump in the first band of the QUEST survey, but only two of the stars identified there as probable clump members are in our sample. The addition of RRLS from the 2nd QUEST band makes the clump even more prominent, and there are 9 stars in our sample that now appear to be outliers of the clump. If the band of stars at $D \sim 8.5 \mathrm{kpc}$ and the $12.4 \mathrm{hr}$ clump are real halo substructures, many of the stars should have similar values of $V_{g s r}$.

Figure 5 is a histogram of $V_{\mathrm{gsr}}$ of the whole sample of RRLS. The mean velocity is $+27 \mathrm{~km} \mathrm{~s}^{-1}$, and the standard deviation is $135 \mathrm{~km} \mathrm{~s}^{-1}$. These values disagree with recent measurements of samples of BHB stars in the halo (see below). For a sample of 1170 BHB stars, Sirko et al. (2004b) found a line-of-sight velocity dispersion, $\sigma_{\text {los }}=101.6 \mathrm{~km} \mathrm{~s}^{-1}$, and $\left\langle V_{\mathrm{gsr}}\right\rangle=0 \mathrm{~km} \mathrm{~s}^{-1}$. Brown et al. (2007) found a similar $\sigma_{\text {los }}$ in their sample of BHB selected from the 2MASS survey, although they noted that the velocity dispersion increased to $117 \mathrm{~km} \mathrm{~s}^{-1}$ if they selected only the most metal poor stars in their sample to avoid thick disk contamination. The Shapiro-Wilk test for normality (Shapiro \& Wilk 1965)1 indicates that the $V_{g s r}$ distribution of our sample does not deviate significantly from a normal distribution. It seems safe, therefore, to use the student t-test and the F-test, which reject with $>90 \%$ confidence that our sample has the same mean velocity and velocity dispersion as the BHB sample of Sirko et al. (2004b). However, the velocity dispersion of our sample is not significantly different from the value Brown et al. (2007) obtained for their most metalpoor BHB stars. While these differences may be another sign that RRLS and BHB stars in the halo have different kinematics (see below and Kinman et al. 2007), the halo substructure in our sample is definitely a major contributor to its relatively large velocity dispersion and its positive mean velocity.

While not statistically different from a normal curve, the histogram in Figure 5 is clearly

\footnotetext{
${ }^{1}$ we used the algorithm by Royston (1995).
} 
unexpected for a random selection of halo objects, for it has a very prominent peak at $V_{g s r} \sim$ $+230 \mathrm{~km} \mathrm{~s}^{-1}$. In total, there are eight RRLS, or $19 \%$ of our sample that have $V_{g s r}>+180$ $\mathrm{km} \mathrm{s}^{-1}$, which is very unlikely to be random occurrence $\left(1\right.$ in 10,000 , if $\sigma_{\text {los }}=101.6 \mathrm{~km} \mathrm{~s}^{-1}$ and $\left.\left\langle V_{\text {gsr }}\right\rangle=0\right)$. This excess of RRLS with large positive $V_{g s r}$ is most likely due to a halo substructure. Other likely substructures, which have less extreme values of Vgsr, are revealed when distance and velocity are used in concert.

In Figure 6, we present histograms of $V_{g s r}$ for these 3 distance intervals, where we have selected dividing lines that avoid putting stars on the boundaries. The histograms in Figure [6 do not resemble the normal curves expected of random selections of halo stars, and in each case the Shapiro-Wilk test rejects normality with greater than $98 \%$ confidence. When considering the $D<6.5 \mathrm{kpc}$ interval, it is important to recall that QUEST survey is probably incomplete at the bright magnitudes of these relatively nearby stars because of saturation of one or more detectors in the QUEST mosaic camera (see Vivas et al. 2004). Detector saturation was not a problem for the stars in the more distant intervals, and the QUEST survey is expected to be $\sim 95 \%$ and $\sim 70 \%$ complete for the type ab and type c variables, respectively. For these intervals at least, the departures of the $V_{\text {grs }}$ distributions from the expected halo curves are therefore probably due the presence of halo substructure.

\subsection{Identification of Groups}

To identify possible groups of stars, we implemented with only a few modifications the algorithm described by Clewley \& Kinman (2006) (the Stellar Pair Search). In this technique, the distances of each star from all others in the sample are calculated from the cartesian Galactic coordinates $x, y$ and $z$. Pairs of stars are ones that are separated by less than a critical distance, $d_{c r i t}$ and have a difference in radial velociticy less than a critical velocity, $v_{\text {crit }}$. Pairs having one star in common form a group. A group may be formed by many connected pairs, and the minimum number of stars in a group, $N$, is 3 (two pairs connected by one star).

To study the statistical significance of the groups we performed Monte Carlo simulations of random samples of stars having the radial distribution of the RRLS in the halo. Specifically, we used the density radial profile described in Vivas \& Zinn (2006) for the direction $l=300^{\circ}, b=60^{\circ}$, which has a slope of -3.1 and takes into account the flattening of the inner halo. We assigned to each simulated star a random $\alpha$ and $\delta$ within the same limits of our sample, and a random $V_{\text {gsr }}$ drawn from a Gaussian distribution with $\sigma_{\text {los }}=101.6 \mathrm{~km} \mathrm{~s}^{-1}$ and $\left\langle V_{\mathrm{gsr}}\right\rangle=0$. Each simulated sample was introduced in the group detection algorithm described above. The experiment was repeated many times, and we counted how many times 
the simulated samples produced groups with characteristics similar to the groups in the real sample of RRLS.

The choices for the parameters $d_{\text {crit }}$ and $v_{\text {crit }}$ require some discussion. As we described above, the VOD covers the whole range of right ascension of the sample studied here, which is $\sim 40^{\circ}$. Any substructure in our data may be as large as this. At $7 \mathrm{kpc}$ from the Sun, $40^{\circ}$ is equivalent to $5 \mathrm{kpc}$, and at $12 \mathrm{kpc}$ from the Sun, it is $8 \mathrm{kpc}$. On the other hand, the uncertainty in distance of the RRLS is $\sim 7 \%$ (Vivas et al. 2004), which suggests a lower limit of $d_{\text {crit }} \lesssim 0.8 \mathrm{kpc}$. The streams from either destroyed globular clusters or dwarf galaxies are expected to be cold substructures, with velocity dispersion $\lesssim 30 \mathrm{~km} \mathrm{~s}^{-1}$. Given the errors in $V_{\gamma}, v_{\text {crit }}$ should be larger than $17 \mathrm{~km} \mathrm{~s}^{-1}$. Since halo substructures are unlikely to have uniform sizes or velocity dispersions, we ran the group detection algorithm and the Monte Carlo simulations using combinations of $d_{\text {crit }}$ from 1 to $4 \mathrm{kpc}$ in steps of $0.5 \mathrm{kpc}$ and $v_{\text {crit }}$ from 20 to $30 \mathrm{~km} \mathrm{~s}^{-1}$ in steps of $5 \mathrm{~km} \mathrm{~s}^{-1}$. A group in the RRLS data was only considered real when less than $5 \%$ of the 5000 Monte Carlo simulations (a $2 \sigma$ detection) produced a group with $\geq N$ members within $\pm 20 \mathrm{~km} \mathrm{~s}^{-1}$ of $\left|V_{g s r}\right|$, the absolute value of the mean velocity of the group of real RRLS. The simulations therefore counted random groups near the $V_{g s r}$ of the suspected group in the real data and ones near $-V_{g s r}$. If a group was detected with different sets of parameters, as happened in most cases, we report here the parameters that gave the lowest probability of random occurrence and/or the maximim number of group members. We restricted our sample to the 33 stars that have $D \geq 6.5 \mathrm{kpc}$ since the incompleteness that is caused by the different saturation levels of the CCDs of the QUEST camera (Vivas et al. 2004) may effect the interpretation of results at closer distances.

Two significant groups were found among our RRLS (Figure 7). As we suspected from the histograms in Figures 5 and 6, there is a group with large positive velocity in our data. The maximum number of members (8 RRLS) was obtained with $d_{\text {crit }}=3.5 \mathrm{kpc}$ and $v_{\text {crit }}=25$ $\mathrm{km} \mathrm{s}^{-1}$. Not one of the 5000 Monte Carlo simulations produced a similar group. The stars making up this group are spread in $D$ from $\sim 7.5$ to $\sim 12.5 \mathrm{kpc}$ (see Figure 7 ). Four of the more distant stars in this group $(D>11 \mathrm{kpc})$ are part of the near side of the " $12.4 \mathrm{hr}$ clump". The unusually high velocities of these stars and that the fact that some of them are probably part of the "12.4 hr clump", unquestionably an over-density in RRLS, suggest that they constitute a halo substructure that may be part of the VOD (see $\S$ - $)$. The combination of large $V_{g s r}$ and a spread in $D$ is consistent with a stellar stream that obliquely cuts the line-of-sight (see Fig. 15 in Harding et al. 2001).

The other significant group contains 9 RRLS. While it is detected with several choices for $d_{\text {crit }}$ and $v_{\text {crit }}$, the values of $1.5 \mathrm{kpc}$ and $25 \mathrm{~km} \mathrm{~s}^{-1}$, respectively, yield the lowest probability of a random occurrence (only $0.7 \%$ of the Monte Carlo simulations produced similar groups, 
a $2.7 \sigma$ detection). The relatively small value of $d_{\text {crit }}$ of this group indicates that it is more concentrated in space than the high velocity group (see Figure 7). It lies $\sim 8.5 \mathrm{kpc}$ from the Sun and has a mean $V_{g s r}$ of $-56 \mathrm{~km} \mathrm{~s}^{-1}$. This group may be due to a stellar stream that cuts the line-of-sight at nearly a right angle, which would explain the narrow distribution in $D$ and the relatively low $V_{g s r}$ of the stars.

\subsection{Comparison with Blue Horizontal Branch Stars}

If any of the features described above are real substructures, then they should also appear in the distributions of the other types of stars that make up very old stellar populations. While BHB stars and RRLS are, of course, samples of horizontal branch stars that differ primarily in temperature, there are good reasons to suspect that they may not always trace the same stellar populations. The majority of the globular clusters in the outer halo have red HBs, while the metal-poor globular clusters in the inner halo have primarily blue HBs (the dependence of the second parameter of HB morphology on galactocentric distance). Consequently, there are large differences in the frequencies of RRLS and BHB stars between the clusters in the inner and outer halos (Suntzeff et al. 1991). The majority of the dwarf spheroidal satellite galaxies of the Milky Way and M31 have red HBs and high frequencies of RRLS and low frequencies of BHB stars (Vivas \& Zinn 2006). Kinman et al. (2007) have suggested that this variation in the RRLS/BHB star ratio and the kinematic disconnect between the inner and outer halos (see $\S$ 1) may be responsible for the kinematic differences that they found between samples of RRLS and BHB in the same region of the Galactic halo. These observations and the trend in RRLS/BHB suggest that the RRLS may be a denser tracer of substructure in the outer halo than BHB stars. Since a small fraction of outer halo globular clusters and dSph galaxies have blue HBs, each with minority populations of RRLS, and since a small population of BHB stars exists in the red HB clusters, some correlation in phase space is expected between these types of stars in the outer halo. On the other hand, an exact correspondence would be surprising.

The sample of BHB stars considered here was drawn primarily from the survey by Sirko et al. (2004a) that is based on photometry and spectroscopy from the SDSS. This database of BHB stars is less complete than our RRLS sample because the SDSS spectroscopy targeted primarily quasars and galaxies. The recent, more complete sample of BHB stars

studied by Brown et al. (2007) unfortunately does not overlap with the Virgo region. From the Sirko et al. (2004a) catalog, we selected all BHB stars (35 in total) within the same range of $\alpha$ as the RRLS sample. In $\delta$ we expanded the limits to $-3.5<\delta<+4$.0. This is still a small range compared with both the width of the region in $\alpha$, and the size of the VOD. We 
also constrained the distance range to $3.0<D<13.0 \mathrm{kpc}$. Sirko et al. estimate that their measurements have an average error in velocity of about $26 \mathrm{~km} \mathrm{~s}^{-1}$.

It has been established both from observations of globular clusters and theoretical modelling that there is a relation between the luminosity of BHB stars and their temperature in the sense that bluer BHB stars are fainter than red BHB stars (see for example Brown et al. 2005). When estimating the distances to their BHB sample, Sirko et al. (2004a) adopted instead the same $L / L_{\odot}$ for all BHB stars on the basis of some observational evidence that they realized was in conflict with theoretical computations. Sirko et al. noted that the adoption of the variation in $L / L_{\odot}$ with effective temperature that is suggested by the computations would lead to smaller distance moduli by an average of 0.18 mag. Kinman et al. (2007) have used the color-magnitude diagrams of globular clusters to derive a relationship between the $B-V$ colors of BHB stars and their $M_{V}$ values, which is anchored on the same $M_{V}$ for RRLS that we are using. A comparison, after making the necessary transformations between photometric systems, of the Kinman et al. (2007) relationship to the one employed by Sirko et al. (2004a) reveals that the difference in distance modulus is near zero at the blue edge of the instability strip and again small at the highest temperatures, but is $0.33 \mathrm{mag}$ (a factor of 1.16 in distance) at $g-r=-0.23$ or $B-V=0$. Because only a fraction of the sample of BHB stars observed by Sirko et al. (2004a) may be effected by distance errors, we have not attempted to recalibrate their distance scale. None of the following comparisons depend critically on an exact match of the BHB and RRLS distance scales.

In a few of the fields that were observed with the Hydra multi-object spectrograph on the WIYN telescope, we identified potential BHB stars from the QUEST photometry. The three stars that listed in Table 4 appear to be true BHB stars. Their $(u-g)_{0}$ and $(g-r)_{0}$ colors, as measured by the SDSS, are consistent with the low gravities of BHB stars on the basis of Figure 10 in Yanny et al. (2000). ¿From our spectra, we measured the full widths of the $\beta, \gamma$, and $\delta$ Balmer lines at 0.2 below the continuum level, which are well-known diagnostics of surface gravity at the temperatures of BHB stars (eg. Wilhelm et al. 1999). In addition to the candidate BHB stars, we measured the WIYN spectra of two BHB stars in the globular cluster M3 that are similar in $(B-V)_{0}$ color to the candidates and of the bright $\mathrm{A}$ and $\mathrm{F}$ type stars that we employed as radial velocity standards with the WIYN observations. The candidate BHB stars have smaller H-line widths than the main-sequence stars and ones that are similar to the BHB stars in M3. To permit direct comparison with the Sirko et al. (2004a) sample of BHB stars, we adopted their method to derive the distances to these BHB stars (see Table 4).

In Figure 8, we have plotted histograms of $V_{g s r}$ for the BHB stars from Sirko et al. (2004a) and Table 4 that lie in the same $D$ intervals as the ones in Figure6. In this diagram, 
there may be more smearing between the intervals than for the RRLS because the absolute magnitudes of the BHB stars are more uncertain (see above). Neither of the two features identified in the RRLS sample is recognizable in the BHB star data alone, which could be due to the incompleteness of the sample or to low frequencies of BHB stars in these groups. However, several BHB stars have $V_{g s r}$ values that coincide with the peaks in the RRLS histogram. Indeed, when we introduced the combined sample of RRLS and BHB stars into the group detection algorithm described above, the same two groups identified in the RRLS sample alone appeared. The group with negative velocities now contains 14 members, 5 of which are BHB stars. The new mean velocity is $-49 \mathrm{~km} \mathrm{~s}^{-1}$, which we adopt as the velocity of the group. Only $5 \%$ of the Monte Carlo simulations produced similar groups. The group with high positive velocities has 10 members (2 BHB stars). Similar groups were obtained in only 2 out of 5000 simulations $(0.04 \%)$.

The combined sample of RRLS and BHB stars also contains a third, small but nonetheless significant, group having a mean radial velocity of $-171 \mathrm{~km} \mathrm{~s}^{-1}$. The group, which was

detected with $d_{\text {crit }}=1 \mathrm{kpc}$ and $v_{\text {crit }}=25 \mathrm{~km} \mathrm{~s}^{-1}$, contains only 3 members, all of which are BHB stars located in the very narrow intervals of $D=11.1$ to $11.3 \mathrm{kpc}$ and $\alpha=205^{\circ}$ to $210^{\circ}$. The Monte Carlo simulations found simialr groups in $\sim 4 \%$ of the cases (a $2.5 \sigma$ detection). This group may be related to main sequence stars with similar velocities (see below) and with 2 RRLS at essentially the same distance and velocity but farther west $\left(\alpha \sim 187^{\circ}\right)$. Since this group lies near the faint limit of our sample, it may be the near side of a larger substructure.

\subsection{Comparison with main-sequence stars}

The recent investigation of the VOD region by Newberg et al. (2007) has provided radial velocity measurements for many stars with the colors of $\mathrm{F}$ type stars in two SEGUE fields, at $(l, b)=\left(288^{\circ}, 62^{\circ}\right)$ and $\left(300^{\circ}, 55^{\circ}\right)$, one of which overlaps with the region we are considering here (see Figure11). Newberg et al. (2007) make the very reasonable assumption that the vast majority of these stars are probably near the main-sequence turnoff in the color-magnitude diagram. Because such stars have a significant spread in absolute magnitude, their distances are much less precisely known than either the RRLS or the BHB stars. The big advantage of using these stars is that they are much more numerous than either RRLS or BHB stars. Below we examine their data for evidence for the major groups described above.

Group at $V_{\boldsymbol{g s r}}=+\mathbf{2 1 5} \mathbf{k m ~ s}^{-\mathbf{1}}$. This group is probably the most remarkable feature seen in our data since very few stars with such high velocities are expected in a sample of 
halo stars (1 or 2 in our sample of RRLS). The 10 RRLS and BHB stars in this group have a mean velocity of $\left\langle V_{\mathrm{gsr}}\right\rangle=+215 \mathrm{~km} \mathrm{~s}^{-1}$, and a standard deviation of $25 \mathrm{~km} \mathrm{~s}^{-1}$, which is not much larger than the typical error in our radial velocities $\left(17 \mathrm{~km} \mathrm{~s}^{-1}\right)$. As noted above, this group appears to be spread along the line of sight, and it may be significant that in the sample of RRLS studied by Duffau et al. (2006), there are 3 stars with $V_{g s r}$ values consistent with membership in this group that have $D$ between 16 and $17 \mathrm{kpc}$.

In their discussion of the F-type stars, Newberg et al. (2007) briefly mention an excess of high velocity stars, which we suspect is related to this group of RRLS and BHB stars. In their Figure 9, Newberg et al. (2007) present for each of their fields histograms of $V_{g s r}$ for the $\mathrm{F}$ stars in two ranges in $g_{0}$ magnitudes. In the histogram for the brighter group, which according to the distance scale2 of Newberg et al. (2007) corresponds to 11 to $14 \mathrm{kpc}$, there are $8 \mathrm{~F}$ type stars with $V_{g s r}$ consistent with assignment to our high velocity group. It is probably significant that all 8 of these stars are found in the more northern of the two fields (see Figure 1), the one that overlaps with the region studied here (2 stars of even higher $V_{g s r}$ are present in this field as well). Since neither one of the two F-star fields has any stars with $V_{g s r}<-200 \mathrm{~km} \mathrm{~s}^{-1}$, there is an asymmetry toward large positive velocities in the same region of the sky where we find one in the RRLS sample. Among the fainter F type stars, which lie at distances between 14 and $18 \mathrm{kpc}$, there are only 3 stars in the 2 fields combined with $V_{g s r}$ within the range of this group.

We have been able to determine metallicities for 6 of the 8 RRLS in our sample that appear to be members of this group. They have a mean $\langle[\mathrm{Fe} / \mathrm{H}]\rangle=-1.55$, with a standard deviation of 0.15 dex, which is of the same size as the uncertainty in our $[\mathrm{Fe} / \mathrm{H}]$ measurements. This narrow distribution is much more consistent with the debris from a destroyed globular cluster than from a dwarf galaxy. Two of the 3 high-velocity RRLS measured by Duffau et al. (2006) have metallicities that are similar to the metallicities of the brighter RRLS. The other one has a much lower $([\mathrm{Fe} / \mathrm{H}]<-1.95)$, which may be a sign that it is unrelated. We are observing more RRLS in the range $12<D<16 \mathrm{kpc}$, with the expectation that more velocity and metallicity measurements will reveal the origin of this substructure.

\footnotetext{
${ }^{2}$ these distance limits and all ones mentioned in connection to the F-type main-sequence stars have substantial uncertainties. The distance scale of Newberg et al. (2007) is based on the luminosity function of Sgr tidal debris (Newberg et al. 2002), which suggests that the assumption of one absolute magnitude for the F-type stars yields a distance uncertainty of $16 \%$. If however F-type stars come from a more heterogeneous population, resembling for example the globular clusters considered by Bell et al. (2007) that span a range in $[\mathrm{Fe} / \mathrm{H}]$, then the distance uncertainty may be as large as $40 \%$
} 
Group at $V_{g s r}=-49 \mathbf{k m ~ s}^{-1}$. This is the most populated group that we find in our data, containing 9 RRLS and 5 BHB stars with velocities in the range $-80<V_{g s r}<-10$ $\mathrm{km} \mathrm{s}^{-1}$. The mean velocity of this group is $\left\langle V_{\mathrm{gsr}}\right\rangle=-49 \mathrm{~km} \mathrm{~s}^{-1}$, with a standard deviation of $22 \mathrm{~km} \mathrm{~s}^{-1}$. The contamination from unrelated halo stars is expected to be relatively high at the low velocity of this group, and assuming the normal distribution in Figure 5 for such field stars, we estimate that only about $50 \%$ of the stars in this velocity interval are probably members of a stream. Thus, our estimates for both the mean velocity and the velocity dispersion are likely to be skewed by this contamination. The 9 RRLS in this group have $\langle[\mathrm{Fe} / \mathrm{H}]\rangle=-1.72$, with a standard deviation of 0.28 dex. There is not a strong peak in the metallicity distribution that would suggest that the large dispersion is caused by contamination by non-members of an otherwise narrow distribution.

Newberg et al. (2007) found a significant peak at $-76 \mathrm{~km} \mathrm{~s}^{-1}$ in the velocities of the F-type stars, which considering the uncertainties is close to the peak that we find. While this suggests membership in the same halo substructure, it is not altogether clear that they overlap spatially. This feature is most clearly evident in the southern of the two fields investigated by Newberg et al. (2007), the one that is outside the region investigated here, and among the bluer and brighter sample of F-type stars $(11 \lesssim D \lesssim 14 \mathrm{kpc}$ if they have $M_{g}=+4.2$ ). On the other hand, the RRLS in this group have $7.5<D<9.5 \mathrm{kpc}$, which place them in the foreground of the F-type stars, unless the F-type stars are less luminous than suspected. There is better spatial coincidence between the F-type stars and a group of fainter RRLS that were measured by Duffau et al. (2006), which have similar $V_{g s r}$. The relationships between the F-type stars, the RRLS in Duffau et al., a sample of M giants, and the tidal stream from the Sgr dwarf galaxy are discussed at length by Newberg et al. (2007), who suspect that this feature may be a new halo substructure that is unrelated to Sgr. The feature that we have identified appears to be unrelated to Sgr as well (see below). More observations are required to clarify its relationship to the feature described by Newberg et al. (2007).

Group at $\boldsymbol{V}_{\boldsymbol{g s r}}=\mathbf{- 1 7 1} \mathbf{\mathbf { k m ~ s }} \mathbf{~}^{\mathbf{1}}$. The 3 BHB stars in the group have basically the same values of $D, 11.2 \mathrm{kpc}$, but given the distance uncertainty a spread of $\sim 2 \mathrm{kpc}$ cannot be excluded. Newberg et al. (2007) identify a peak in their F star data at $V_{g s r}=-168 \mathrm{~km} \mathrm{~s}^{-1}$, and in this case, there are good reasons to believe that the features are the same. A peak is seen in both of their fields, although it is more prominent in the southern field, the one that is outside our region, than in the northern. It is only seen in the brighter $\mathrm{F}$ stars that have $0.2<(g-r)_{0}<0.4$. According to the distance scale of Newberg et al. (2007) these stars lie at $D$ between 11 and $14 \mathrm{kpc}$, which agrees well with the distances of the BHB stars. 


\subsubsection{Does the VSS extends to closer distances?}

The $\mathrm{F}$ star data show a very prominent peak at $130 \mathrm{~km} \mathrm{~s}^{-1}$ in both fields, and Newberg et al. consider this to be the velocity of S297+63-20.5 feature. On the basis of the spatial coincidence between S297+63-20.5 and the VSS and the near coincidence in $V_{g s r}$, they conclude that these features are probably the same or physically associated. However, Newberg et al. (2007) note that the $30 \mathrm{~km} \mathrm{~s}^{-1}$ offset in $V_{g s r}$ between S297+63-20.5 and the VSS requires explanation.

The peak at $130 \mathrm{~km} \mathrm{~s}^{-1}$ is seen in the faintest and the reddest group of $\mathrm{F}$ stars, which have $g_{0}$ between 20 and 20.5. Newberg et al. (2007) note, however, that the most of these stars actually have $g_{0}<20.3$, which corresponds to $D<16.7 \mathrm{kpc}$ on their distance scale. Since the core of the VSS is located at about $19 \mathrm{kpc}$ (Duffau et al. 2006), it is possible that $30 \mathrm{~km} \mathrm{~s}^{-1}$ offset is due to a gradient in $V_{g s r}$ with $D$. Some evidence for this is provided by the data for the brighter and bluer F star data (see Figure 10 in Newberg et al.), which has a prominent peak at $\sim 150 \mathrm{~km} \mathrm{~s}^{-1}$. The histograms in Figures 6 and 8 indicate there are 5 stars (2 RRLS and 3 BHB stars) having a mean velocity of $+143 \mathrm{~km} \mathrm{~s}^{-1}$ which, within uncertainties, is identical to the peak seen in the bright F star data. All 5 RRLS and BHB stars are located near the far distance limit of our sample at $D>11.8 \mathrm{kpc}$ and within the near boundary of the " $12.4 \mathrm{hr}$ clump" of RRLS. The 5 stars are indeed identified as a group by our algorithm (with parameters $d_{c r i t}=2.5 \mathrm{kpc}, v_{\text {crit }}=20 \mathrm{~km} \mathrm{~s}^{-1}$ ), but by itself this group has only low significance because $\sim 25 \%$ of the Monte Carlo simulations produce similar groups. While a chance coincidence cannot be ruled out, the similarity in $\left\langle V_{g s r}\right\rangle$ between this group and the peak in the bright F star data suggests that they are related. The group of RRLS and BHB stars provides a hint that the VSS may extend to distances as short as $\sim 12 \mathrm{kpc}$. A gradient in $V_{g s r}$, with $V_{g s r}$ becoming less positive with increasing $D$, is expected of a stellar stream that obliquely cuts the line of sight (see Harding et al. 2001). It is also possible, however, that observational error and sample selection may also explain the $V_{g s r}$ offset. We are exploring the possible extension of the VSS and the possibility of a gradient by measuring more QUEST RRLS at distances between 13 to $18 \mathrm{kpc}$ (Duffau et al. 2008 , in preparation).

\section{THE RELATIONSHIP TO THE SGR STREAMS}

Soon after the first detections of over-densities of stars in the direction of Virgo, several authors (eg. Majewski et al. 2003; Martínez-Delgado et al. 2004) speculated that they may be debris from the disruption of the Sgr dSph galaxy. Even then there was some evidence to contrary, for Newberg et al. (2002) had noted that the color of the turnoff in S297+63- 
20.5 was redder than the color of the turnoff in the Sgr streams. Duffau et al. (2006) later argued that the Sgr streams were not responsible for the VSS or the majority of the other stars in their sample because models of the streams produced very few stars at the observed positions and the predicted values of $V_{g s r}$ did not match the ones observed. The realization by Jurić et al. (2008) that the VOD has huge dimensions on the sky motivated Martínez-Delgado et al. (2007) to reexamine the Sgr connection. Through detailed modeling of the streams, they showed that ones that assume an oblate shape for the gravitational potential of the Galaxy can explain the VOD as the leading stream from Sgr as it plunges toward the Sun and crosses the galactic plane in the solar neighborhood. This model does not, however, provide a good match to our observations, those of Duffau et al. (2006), and more generally the $12.4 \mathrm{hr}$ clump of RRLS.

In the left-hand panels of Figure 9, we have plotted the RRLS in the first band of the QUEST survey that lie within the $\alpha$ range of our sample. Closed symbols depict the RRLS that have measured velocities from Vivas et al. (2005), Duffau et al. (2006), and the present study. These three datasets cover different range of distances, $>40,16-20$ and $<12.5 \mathrm{kpc}$ respectively. The small crosses are the particles in the stream models of Law et al. (2005) under the assumptions that the potential is oblate, spherical, or prolate. The panels on the right compare the model predictions for $V_{g s r}$ as a function of $D$ with the observed velocities.

The QUEST survey detects the Sgr leading arm at $D \sim 50 \mathrm{kpc}$ in the range $195^{\circ}<\alpha<$ $235^{\circ}$ (see Figure 2 and Vivas et al. 2005) and the $12.4 \mathrm{hr}$ clump in the ranges $12<D<20$ $\mathrm{kpc}$ and $170^{\circ}<\alpha<200^{\circ}$ (see Fig. 12 in Vivas \& Zinn 2006). Note that there is not a blending together of these features (see Figure 2), as one would expect if they are parts of the same stream, but instead they are separated by a region of low density. This is not a selection effect introduced by the observing technique (see Vivas et al. 2004).

If the leading arm crosses the galactic plane near the Sun, then the stream stars at small $D$ will have large, negative values of $V_{g s r}$ because they have fallen from $\sim 50 \mathrm{kpc}$ on orbits that are nearly aligned with the line-of-sight from the Sun. This prediction of large negative velocity at small $D$ and the continuity of the stream are two characteristics of this picture that should not be model dependent. Martínez-Delgado et al. (2007) note that their models reproduce the models of Law et al. (2005), which because they are readily available, we will use here to illustrate the salient points of this picture.

Each of the Law et al. models matches reasonably well the positions and the velocities of the RRLS at $\sim 50 \mathrm{kpc}$. However, both the oblate and the spherical models predict smoothly varying densities of RRLS over wide ranges in $D$, which are in conflict with the observed region of relatively low density of RRLS between 20 and $40 \mathrm{kpc}$ (see also Vivas et al. 2005). These same models predict that $V_{g s r}$ becomes more negative as $D$ decreases and reaches quite 
large $\left|V_{\text {gss }}\right|$ at $D<20 \mathrm{kpc}$. Only one RRLS in our sample and one in the sample observed by Duffau et al. (2006) have values of $V_{g s r}$ that are consistent with these models. Also, none of the BHB stars in the region have highly negative velocities (see Figure 8). In the prolate model, the leading stream crosses the galactic plane far from the Sun, which explains why it predicts relatively few stars in the diagrams at $D<20 \mathrm{kpc}$. We conclude on the basis of these comparisons that none of the halo substructures that we have detected are probably due to the leading arm. Moreover, based on the current Sgr models, our observations imply that the shape of the Galactic potential can be neither oblate nor spherical.

Martínez-Delgado et al. (2007) note that in their oblate model the trailing stream of Sgr crosses through the region of the VSS and coincides with the VSS in $V_{g s r}(\sim 90 \mathrm{~km} / \mathrm{s})$. This is a plausible explanation for the VSS, if it is still viable after the oblate model is reconciled with the observations presented here and with other recent observations which indicate that the leading arm does not cross the galactic plane in the solar neighborhood Newberg et al. 2007; Seabroke et al. 2008).

\section{DISCUSSION}

Kinematical information is crucial to detect substructures in regions where no obvious spatial overdensities are present. Taken together, our RRLS sample, the BHB star sample of Sirko et al. (2004a), and the F star sample of Newberg et al. (2007) paint a consistent picture that several separate halo substructures exist at $D \lesssim 13 \mathrm{kpc}$ in the part of the VOD that is covered by the QUEST survey. The RRLS sample of Duffau et al. (2006) and the same BHB and F star samples show that some of these substructures extend to larger $D$. None of these substructures seem to be related with the Sgr leading tail.

The analysis of the SDSS photometric data by Newberg et al. (2002, 2007) make a compelling case that the region of highest stellar density in S297+63-20.5 lies at $D \sim 18$ kpc. This is identical to within the errors of the region of highest density in the $12.4 \mathrm{hr}$ clump of QUEST RRLS, which is where Duffau et al. (2006) discovered the VSS in velocity space. The near coincidence in $\left\langle V_{g s r}\right\rangle$ between the VSS and the largest peak in $V_{g s r}$ that Newberg et al. (2007) observed in S297+63-20.5 provides strong evidence that they are the same. As noted by Newberg et al. (2007), the small offset $\left(\sim 30 \mathrm{~km} \mathrm{~s}^{-1}\right)$ could be due to measurement error and/or by the small sizes of the samples of spectroscopically observed stars. It is also possible that it is due to a gradient in $V_{g s r}$ with $D$ (see above). Newberg et al. (2007) found the same velocity peak in their two spectroscopic fields, which indicates that the S297+63-20.5/ VSS has considerable angular extent on the sky. Other evidence for this is provided by the recent RRLS surveys by Keller et al. (2007) and Wilhelm et al. (2007). 
Keller et al. (2007) found 2 clumps of RRLS south of the VOD region (see Figure 11) at coordinates $(l, b)=\left(301^{\circ}, 56^{\circ}\right)$ and $\left(324^{\circ}, 49^{\circ}\right)$ and distances 16 and $19 \mathrm{kpc}$ respectively. Velocity information is not yet available for these clumps. Wilhelm et al. (2007) identified an excess of candidate RRLS having $V_{g s r} \sim 100 \mathrm{~km} \mathrm{~s}^{-1}$ in the North Galactic Cap $\left(b>70^{\circ}\right)$. The results presented here indicate that the S297+63-20.5/VSS feature may also extend along the line of sight to distances as close as $\sim 11.8 \mathrm{kpc}$.

We conclude that the VOD contains at least one relatively large structure, S297+63-20.5 /VSS, which is intersperced with smaller halo substructures that are unrelated to S297+6320.5/VSS or to each other. While these smaller structures might be peculiar to the VOD region, it is more likely that they are common in the outer halo. They are better documented in the VOD direction simply because the presence of S297+63-20.5/ VSS has drawn attention to this region. In the case of our sample of RRLS, the spatial distribution of the stars

provided only very marginal evidence of any substructure (Vivas \& Zinn 2006). The picture changed dramatically when we used spatial and velocity data together. There is already strong evidence from the spatial distribution of stars in the SDSS that much, if not all, of the outer halo was accreted from the destroyed of satellite galaxies (Bell et al. 2007). Our investigation of the VOD region suggests that the addition of velocity data will make the case even stronger.

This research was supported by the National Science Foundation under grants AST 00-98428 and AST 05-07364. The availability of the SMARTS $1.5 \mathrm{~m}$ telescope and its service observing mode made this project feasible. We thank Charles Bailyn for his efforts to setup the SMARTS consortium and Fred Walter and Suzanne Tourtellotte for their assistance with SMARTS operations and data handling. We are indebted to the service mode observers Sergio Gonzalez, Alberto Pasten, Claudio Aguilera and Alberto Miranda for their fine operation of the telescope and the spectrograph. We also thank the anonymous referee for helpful suggestions to the manuscript.

\section{REFERENCES}

Bell, E. F. et al. 2007, ArXiv e-prints, 0706.0004

Belokurov, V. et al. 2006, ApJ, 642, L137

Brown, W. R., Geller, M. J., Kenyon, S. J., Kurtz, M. J., Allende Prieto, C., Beers, T. C., \& Wilhelm, R. 2005, AJ, 130, 1097 
Brown, W. R., Beers, T. C., Wilhelm, R., Allende Prieto, C., Geller, M. J., Kenyon, S. J., \& Kurtz, M. J. 2007, ArXiv e-prints, 0711.2886

Bullock, J. S., \& Johnston, K. V. 2005, ApJ, 635, 931

Carney, B. W., Latham, D. W., \& Laird, J. B. 1990, AJ, 99, 572

Carollo, D. et al. 2007, Nature, 450, 1020

Chiba, M., \& Beers, T. C. 2000, AJ, 119, 2843

Clewley, L., \& Kinman, T. D. 2006, MNRAS, 371, L11

Duffau, S., Zinn, R., Vivas, A. K., Carraro, G., Méndez, R. A., Winnick, R., \& Gallart, C. 2006, ApJ, 636, L97

Fardal, M. A., Guhathakurta, P., Babul, A., \& McConnachie, A. W. 2007, MNRAS, 380, 15

Ferguson, A. M. N., Irwin, M. J., Ibata, R. A., Lewis, G. F., \& Tanvir, N. R. 2002, AJ, 124, 1452

Freeman, K. C., \& Rodgers, A. W. 1975, ApJ, 201, L71

Freeman, K., \& Bland-Hawthorn, J. 2002, ARA\&A, 40, 487

Gratton, R. G., Bragaglia, A., Clementini, G., Carretta, E., Di Fabrizio, L., Maio, M., \& Taribello, E. 2004, A\&A, 421, 937

Harding, P., Morrison, H. L., Olszewski, E. W., Arabadjis, J., Mateo, M., Dohm-Palmer, R. C., Freeman, K. C., \& Norris, J. E. 2001, AJ, 122, 1397

Hartwick, F. D. A. 1987, in NATO ASIC Proc. 207: The Galaxy, ed. G. Gilmore \& B. Carswell, 281-290

Ibata, R., Irwin, M., Lewis, G., Ferguson, A. M. N., \& Tanvir, N. 2001a, Nature, 412, 49

Ibata, R., Lewis, G. F., Irwin, M., Totten, E., \& Quinn, T. 2001b, ApJ, 551, 294

Ibata, R. A., Irwin, M. J., Lewis, G. F., Ferguson, A. M. N., \& Tanvir, N. 2003, MNRAS, 340, L21

Ivezić, Ž. et al. 2000, AJ, 120, 963

Jurić, M. et al. 2005, ArXiv Astrophysics e-prints, astro-ph/0510520 
—. 2008, ApJ, 673, 864

Kalirai, J. S., Guhathakurta, P., Gilbert, K. M., Reitzel, D. B., Majewski, S. R., Rich, R. M., \& Cooper, M. C. 2006, ApJ, 641, 268

Keller, S. C., Murphy, S., Prior, S., DaCosta, G., \& Schmidt, B. 2007, ArXiv e-prints, 0711.2337

Kemper, E. 1982, AJ, 87, 1395

Kinman, T. D., Cacciari, C., Bragaglia, A., Buzzoni, A., \& Spagna, A. 2007, MNRAS, 375, 1381

Law, D. R., Johnston, K. V., \& Majewski, S. R. 2005, ApJ, 619, 807

Layden, A. C. 1994, AJ, 108, 1016

Lee, Y.-W., Gim, H. B., \& Casetti-Dinescu, D. I. 2007, ApJ, 661, L49

Mackey, A. D., \& Gilmore, G. F. 2004, MNRAS, 355, 504

Majewski, S. R. 1992, ApJS, 78, 87

-. 1993, ARA\&A, 31, 575

Majewski, S. R., Skrutskie, M. F., Weinberg, M. D., \& Ostheimer, J. C. 2003, ApJ, 599, 1082

Martínez-Delgado, D., Gómez-Flechoso, M. Á., Aparicio, A., \& Carrera, R. 2004, ApJ, 601, 242

Martínez-Delgado, D., Peñarrubia, J., Jurić, M., Alfaro, E. J., \& Ivezić, Z. 2007, ApJ, 660, 1264

Miceli, A. et al. 2007, ArXiv e-prints, 0706.1583

Morrison, H. L., Harding, P., Hurley-Keller, D., \& Jacoby, G. 2003, ApJ, 596, L183

Newberg, H. J. et al. 2002, ApJ, 569, 245

Newberg, H. J., Yanny, B., Cole, N., Beers, T. C., Re Fiorentin, P., Schneider, D. P., \& Wilhelm, R. 2007, ApJ, 668, 221

Norris, J. 1986, ApJS, 61, 667 
Oke, J. B., Giver, L. P., \& Searle, L. 1962, ApJ, 136, 393

Oke, J. B. 1966, ApJ, 145, 468

Preston, G. W. 1959, ApJ, 130, 507

Rocha-Pinto, H. J., Majewski, S. R., Skrutskie, M. F., Crane, J. D., \& Patterson, R. J. 2004, ApJ, 615, 732

Royston, P. 1995, Applied Statistics, 44, 547

Sandstrom, K., Pilachowski, C. A., \& Saha, A. 2001, AJ, 122, 3212

Seabroke, G. M. et al. 2008, MNRAS, 58

Searle, L., \& Zinn, R. 1978, ApJ, 225, 357

Shapiro, S. S., \& Wilk, M. B. 1965, Biometrika, 52, 591

Sirko, E. et al. 2004a, AJ, 127, 899

-. 2004b, AJ, 127, 914

Smith, H. A. 1995, RR Lyrae stars (Cambridge Astrophysics Series, Cambridge, New York: Cambridge University Press, —c1995)

Sneden, C., Kraft, R. P., Guhathakurta, P., Peterson, R. C., \& Fulbright, J. P. 2004, AJ, 127,2162

Storm, J., Carney, B. W., Latham, D. W., Davis, R. J., \& Laird, J. B. 1992, PASP, 104, 168

Suntzeff, N. B., Kinman, T. D., \& Kraft, R. P. 1991, ApJ, 367, 528

Vivas, A. K. 2002, PhD thesis, AA(YALE UNIVERSITY)

Vivas, A. K. et al. 2001, ApJ, 554, L33

Vivas, A. K., \& Zinn, R. 2003, Memorie della Societa Astronomica Italiana, 74, 928

Vivas, A. K. et al. 2004, AJ, 127, 1158

Vivas, A. K., Zinn, R., \& Gallart, C. 2005, AJ, 129, 189

Vivas, A. K., \& Zinn, R. 2006, AJ, 132, 714 
Wilhelm, R., Beers, T. C., Sommer-Larsen, J., Pier, J. R., Layden, A. C., Flynn, C., Rossi, S., \& Christensen, P. R. 1999, AJ, 117, 2329

Wilhelm, R. et al. 2007, ArXiv e-prints, 0712.0776

Yanny, B. et al. 2000, ApJ, 540, 825

-. 2003, ApJ, 588, 824

Zinn, R. 1993, in Astronomical Society of the Pacific Conference Series, Vol. 48, The Globular Cluster-Galaxy Connection, ed. G. H. Smith \& J. P. Brodie, 38

Zinn, R., \& West, M. J. 1984, ApJS, 55, 45

Zinn, R., Vivas, A. K., Gallart, C., \& Winnick, R. 2004, in Astronomical Society of the Pacific Conference Series, Vol. 327, Satellites and Tidal Streams, ed. F. Prada, D. Martinez Delgado, \& T. J. Mahoney, 92

Zucker, D. B. et al. 2004, ApJ, 612, L117 


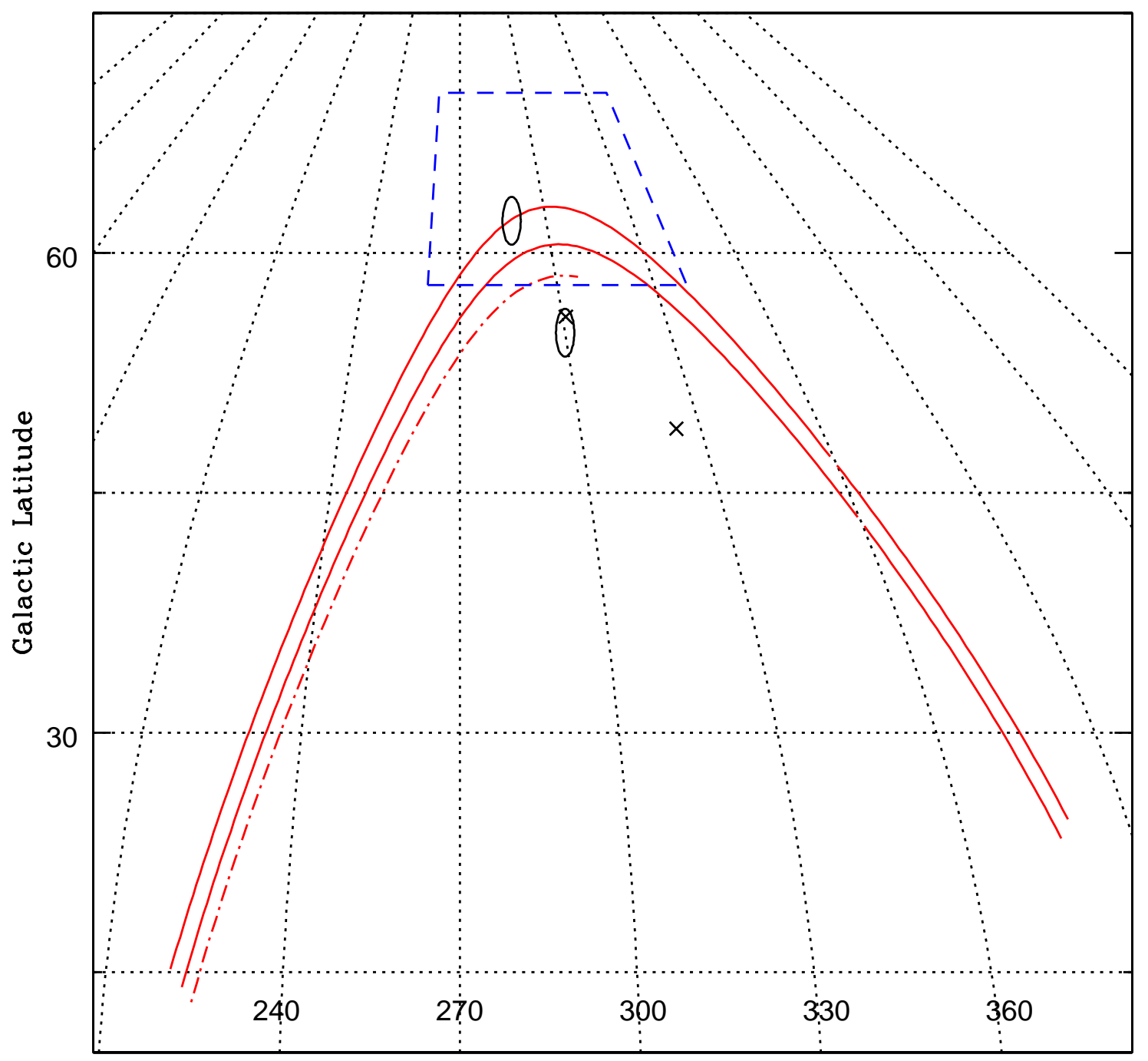

Galactic Longitude

Fig. 1. - Map in Galactic coordinates showing the strip (solid lines) surveyed in the first QUEST catalog in the Northern Galactic Hemisphere. The dot-dashed line indicates the region of the second (unpublished) QUEST catalog. According to Juric et al. (2008), the region of the VOD is roughly enclosed by the polygon. The small ovals indicate the two regions where Newberg et al. (2007) looked for radial velocity substructures among F stars $\left((l, b)=\left(300^{\circ}, 55^{\circ}\right)\right.$ and $\left.\left(288^{\circ}, 62^{\circ}\right)\right)$. The two $\times$ symbols indicate the locations where Keller et al. (2007) found overdensities of RRLS at 16 and $19 \mathrm{kpc}$ from the Sun. 


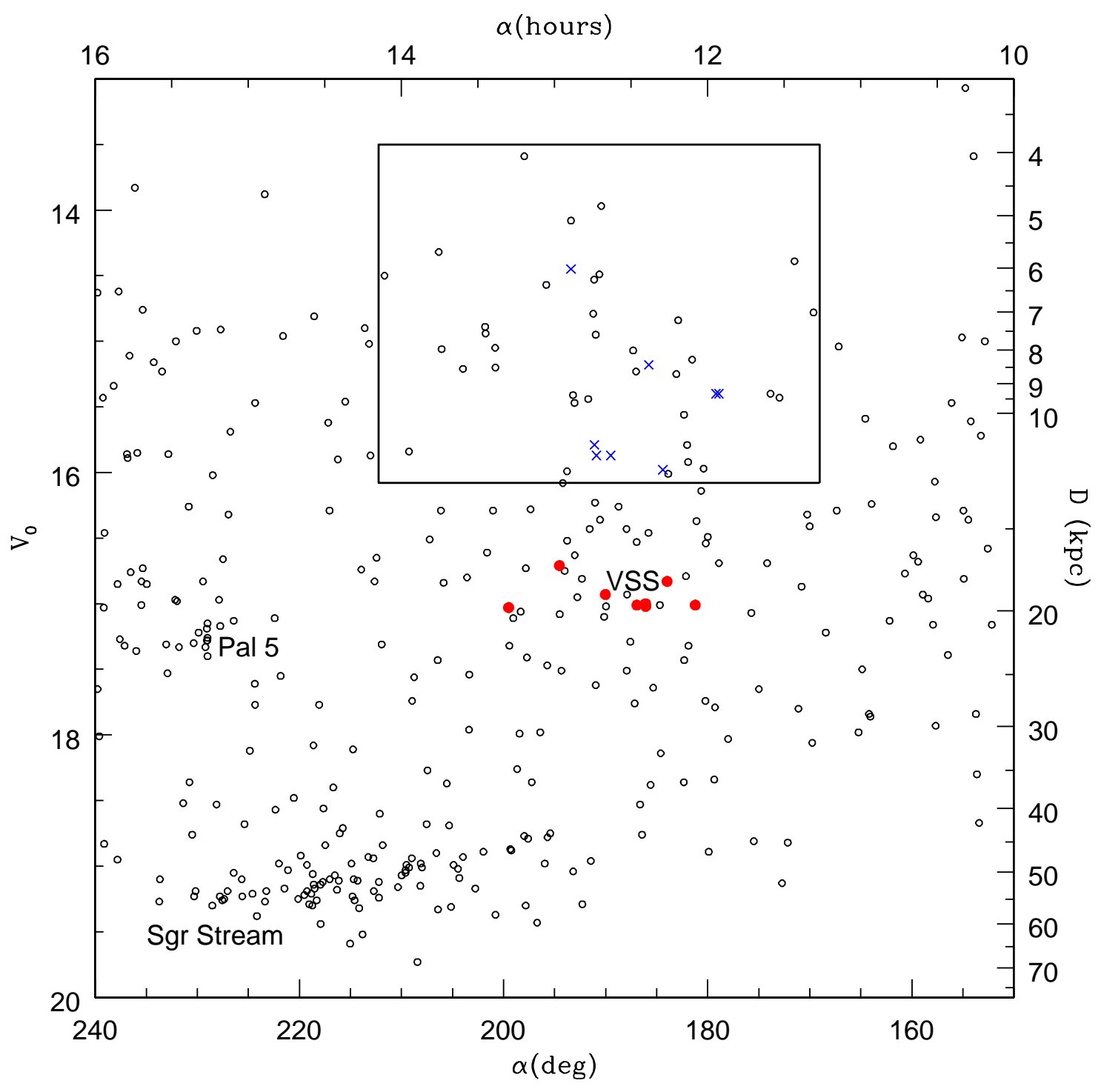

Fig. 2.- Spatial distribution of the QUEST RRLS in the range of right ascension from $150^{\circ}$ to $240^{\circ}$ (from Vivas et al. 2004). The box encloses the bright subsample $\left(V_{0}<16.1\right)$ toward Virgo for which we obtained spectroscopy. The $\times$ symbols indicate the 8 RRLS from the second QUEST catalog with available spectroscopy that are included in our analysis. The large spatial substructures found among the fainter RRLS (see Vivas \& Zinn 2006) are indicated: the Sgr stream, the globular cluster Pal 5 and its tails, and the " 12 h 4 clump", which contains the VSS. We have indicated with solid circles the RRLS having velocities consistent with the VSS (Duffau et al. 2006). 


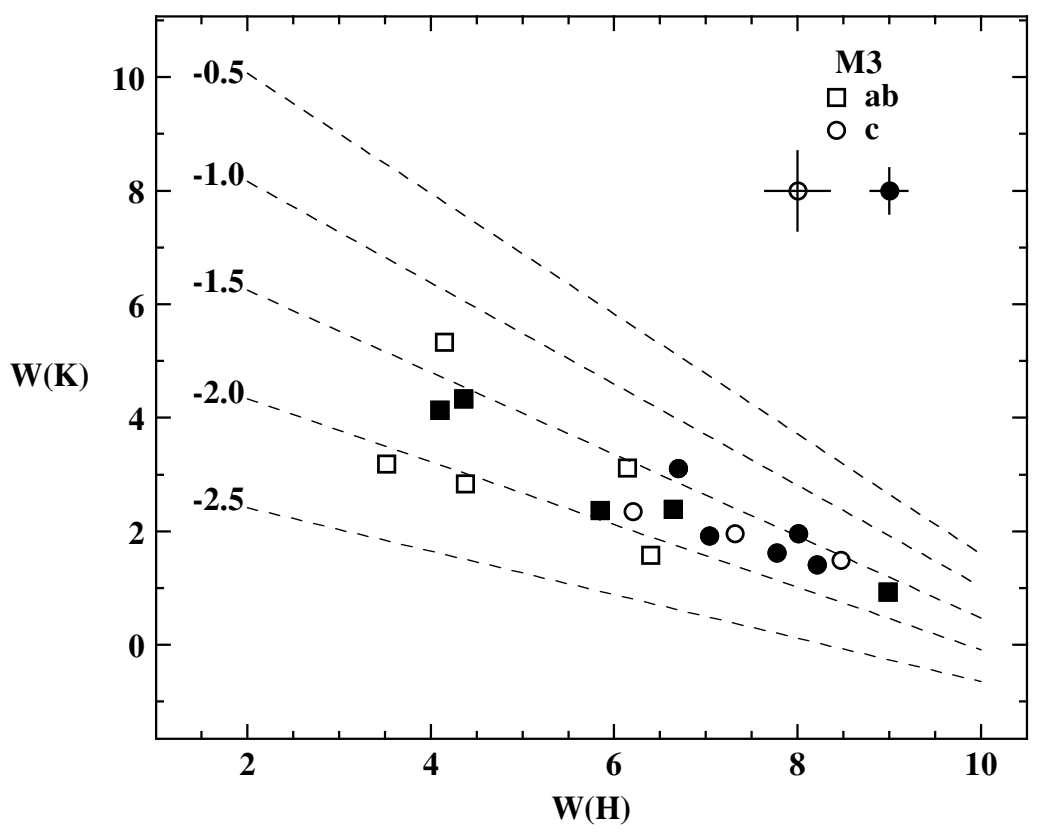

Fig. 3.- Calibration in the plane $\mathrm{W}(\mathrm{K})$ vs $\mathrm{W}(\mathrm{H})$ of the metallicity of RRLS, from Layden (1994). Depending on their metallicity, RR $a b$ stars lie along one of the dashed lines during the pulsation cycle (excluding measurements during the rising branch). Symbols represent measurements of RRLS variables in M3, with filled symbols corresponding to high $\mathrm{S} / \mathrm{N}$ observations. Typical error bars for the low and high $\mathrm{S} / \mathrm{N}$ spectrograms are shown. This diagram shows that Layden's calibration is also valid for $\mathrm{RR} c$ stars. 

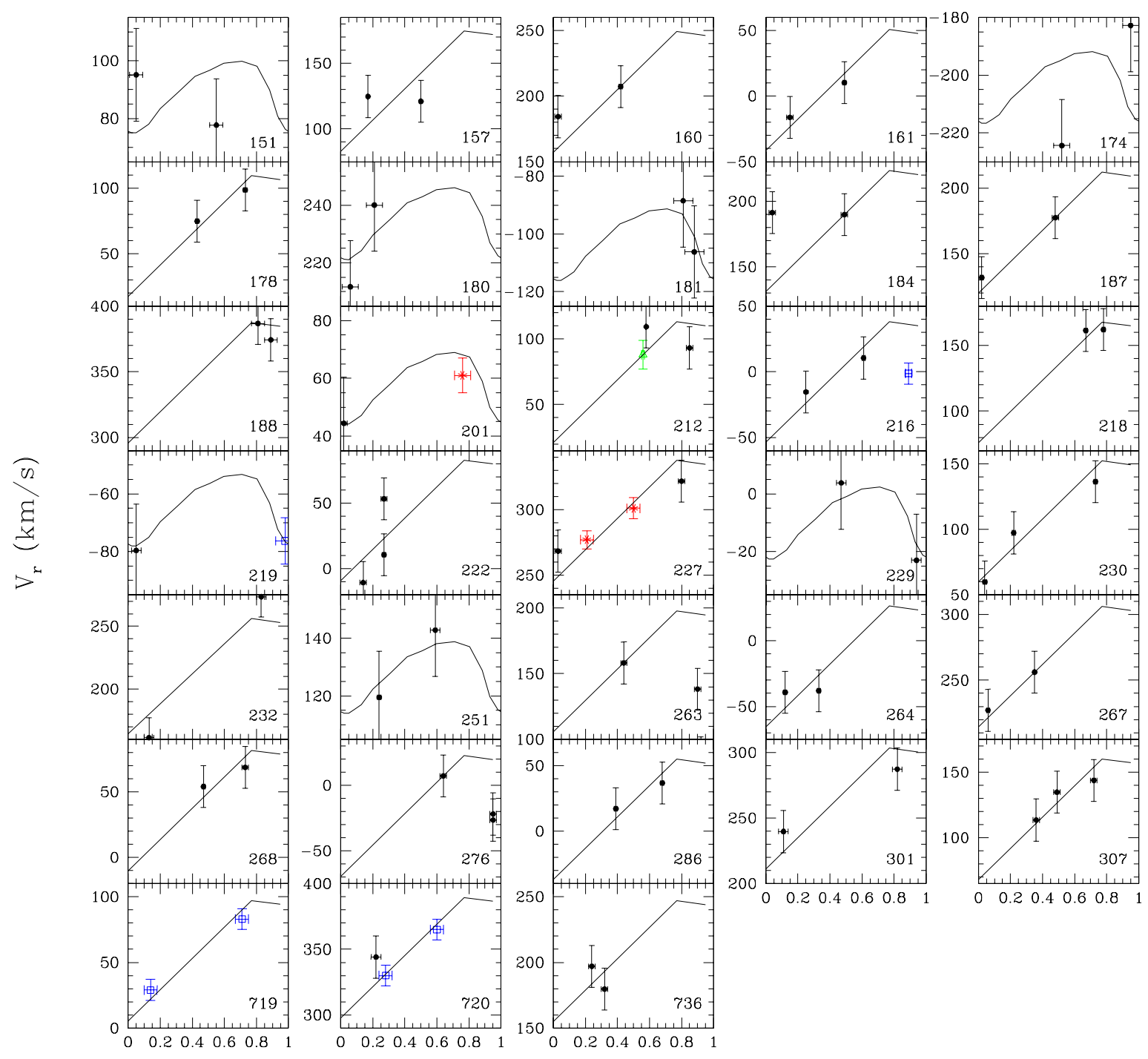

Phase

Fig. 4.- Radial velocity curves for the 33 stars having two or more spectroscopic observations. The SMARTS observations are indicated with solid circles, the ESO observation is marked with an open triangle, the WIYN-R observations are asterisks, and the WIYN-B are open squares. The fitted radial velocity curves are indicated by solid lines. All observations are shown including those ones not used in the fitting of the radial velocity curve because they were taken near the phase of maximum light (see text). Although they were not used in the fitting, they lie within the expected range of velocity. 


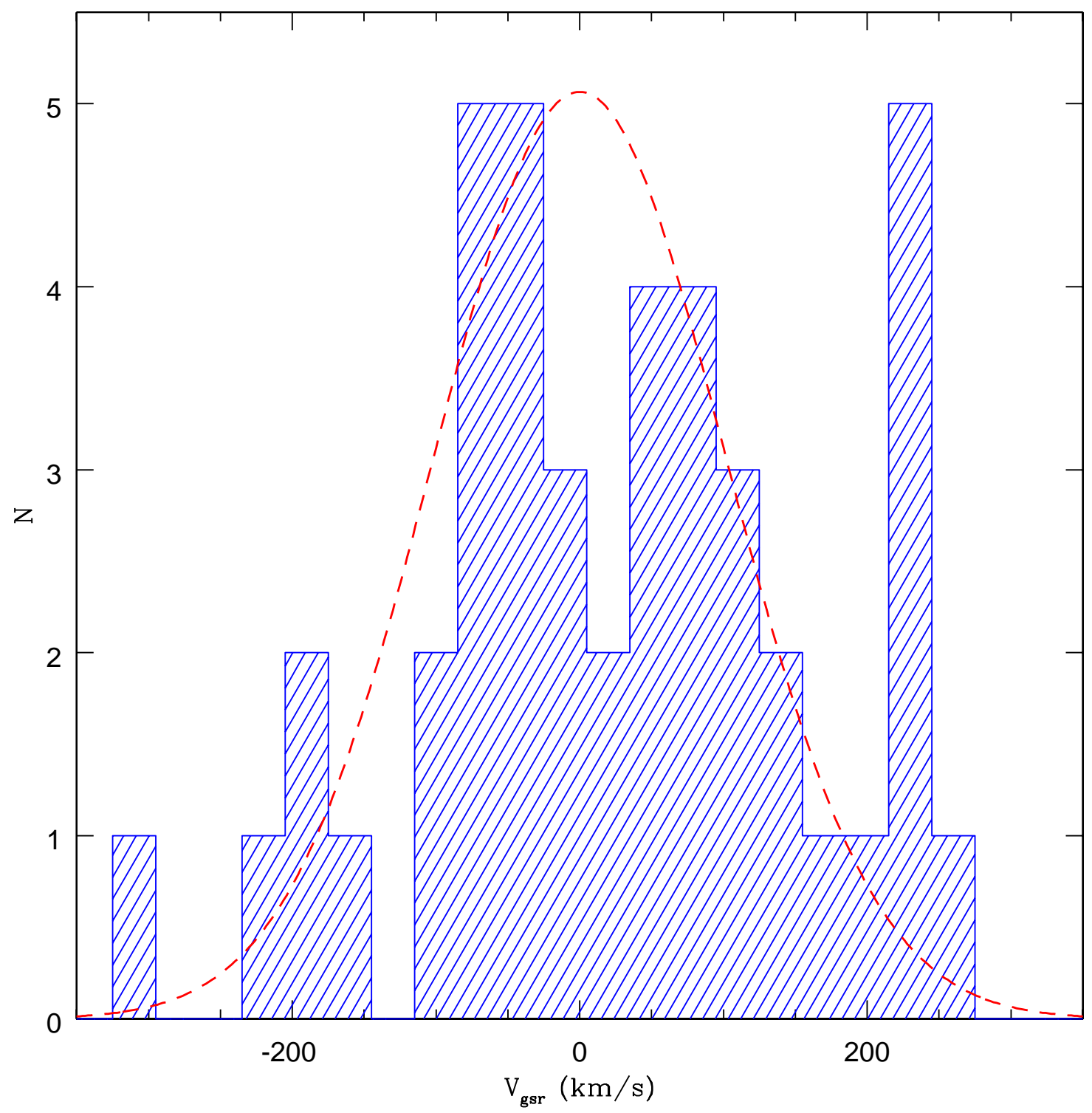

Fig. 5.- Histogram of $V_{g s r}$ of the 43 RRLS. For reference, the dashed line indicates the expected distribution of velocities in a random halo sample $\left(\sigma_{\text {halo }}=101.6 \mathrm{~km} \mathrm{~s}^{-1}\right)$. The size of each bin is $30 \mathrm{~km} \mathrm{~s}^{-1}$. 


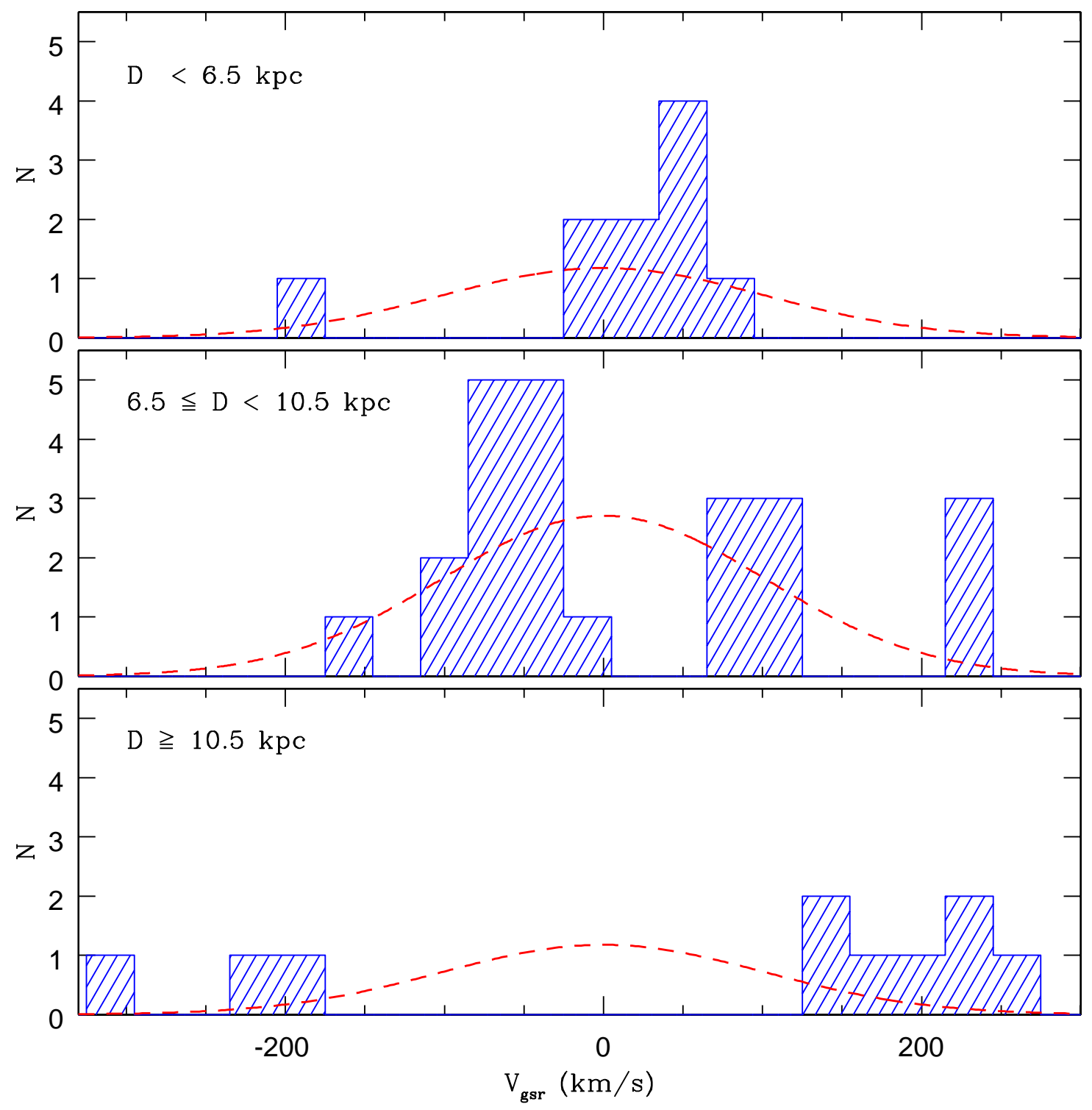

Fig. 6.- Histogram of $V_{g s r}$ of the RRLS in 3 distance bins. The dashed line indicates the expected distribution of velocities in the halo, normalized to the number of stars in each range of distance. 


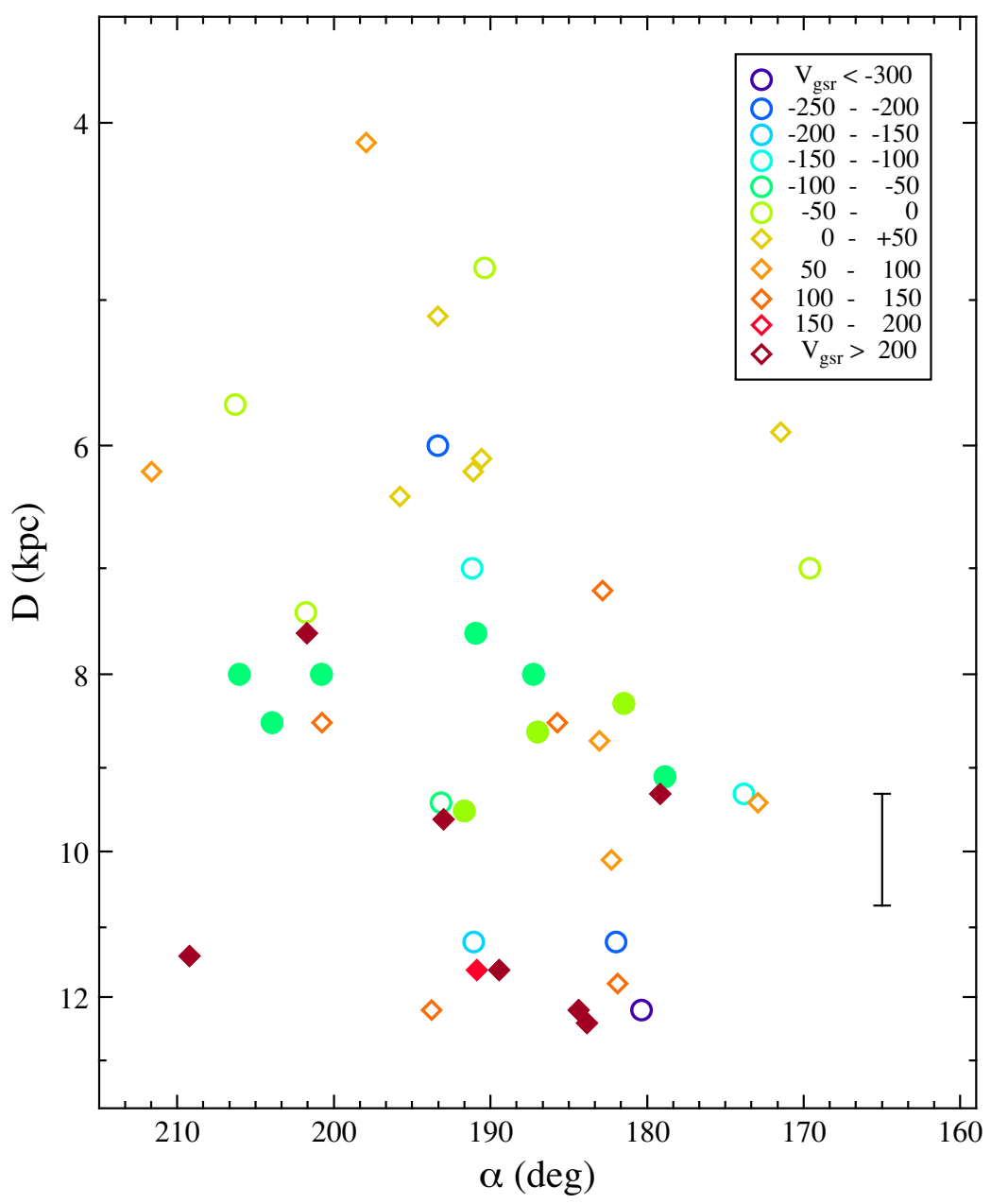

Fig. 7.- Distribution of distance from the Sun of the sample of RRLS as a function of right ascension. Circles and diamond symbols represent stars with negative and positive radial velocities respectively. Colors indicate stars with similar velocities in bins of $50 \mathrm{~km} \mathrm{~s}^{-1}$. The two significant groups found in our data are indicated by solid symbols, one of them have stars with very high positive velocities and the other one has stars with velocities around $-60 \mathrm{~km} \mathrm{~s}^{-1}$. 


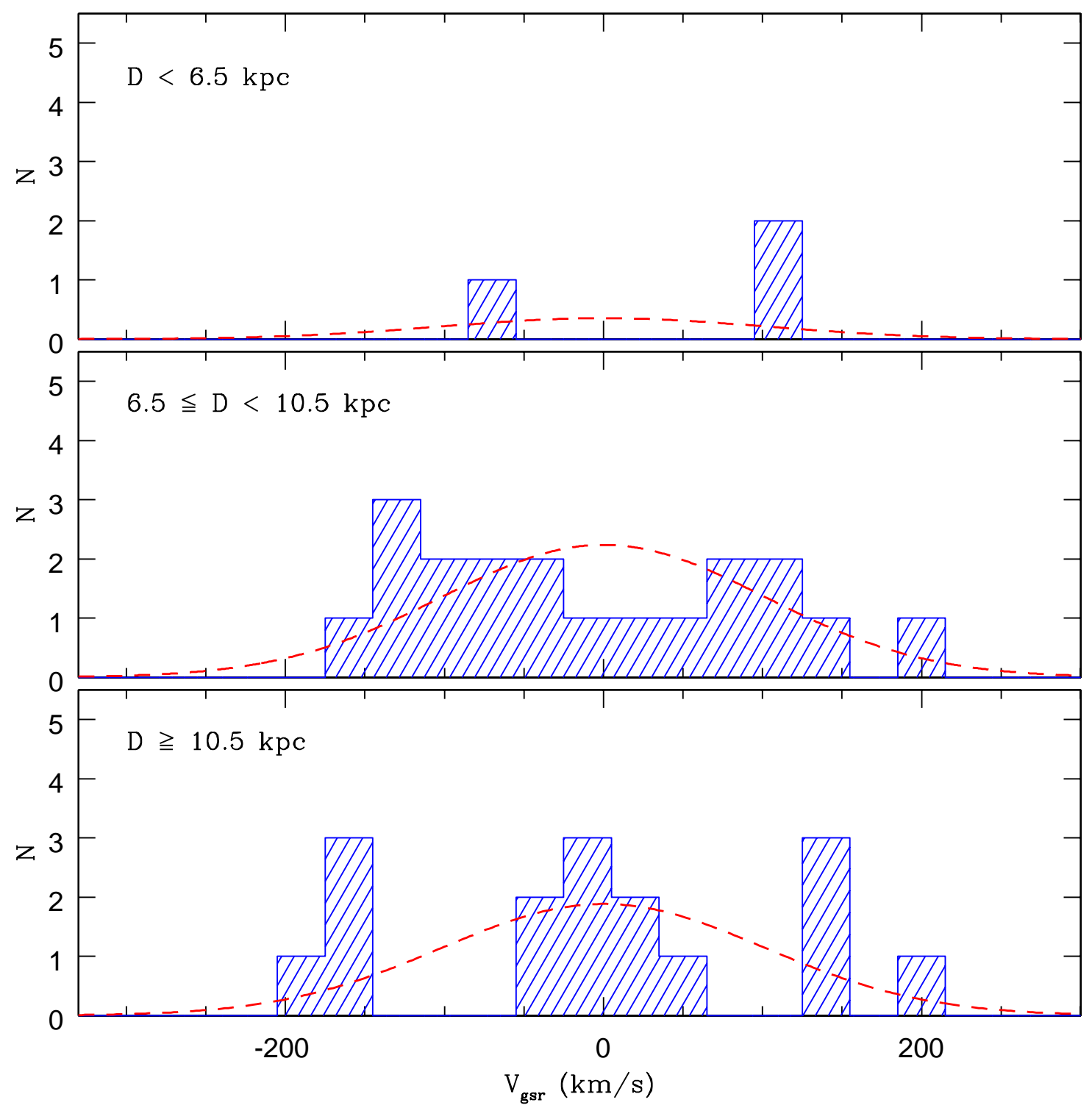

Fig. 8. - Histogram of $V_{g s r}$ of the BHB stars in the same 3 distance bins as Figure 6. The dashed line indicates the expected distribution of velocities in the halo, normalized to the number of stars in each range of distance. 

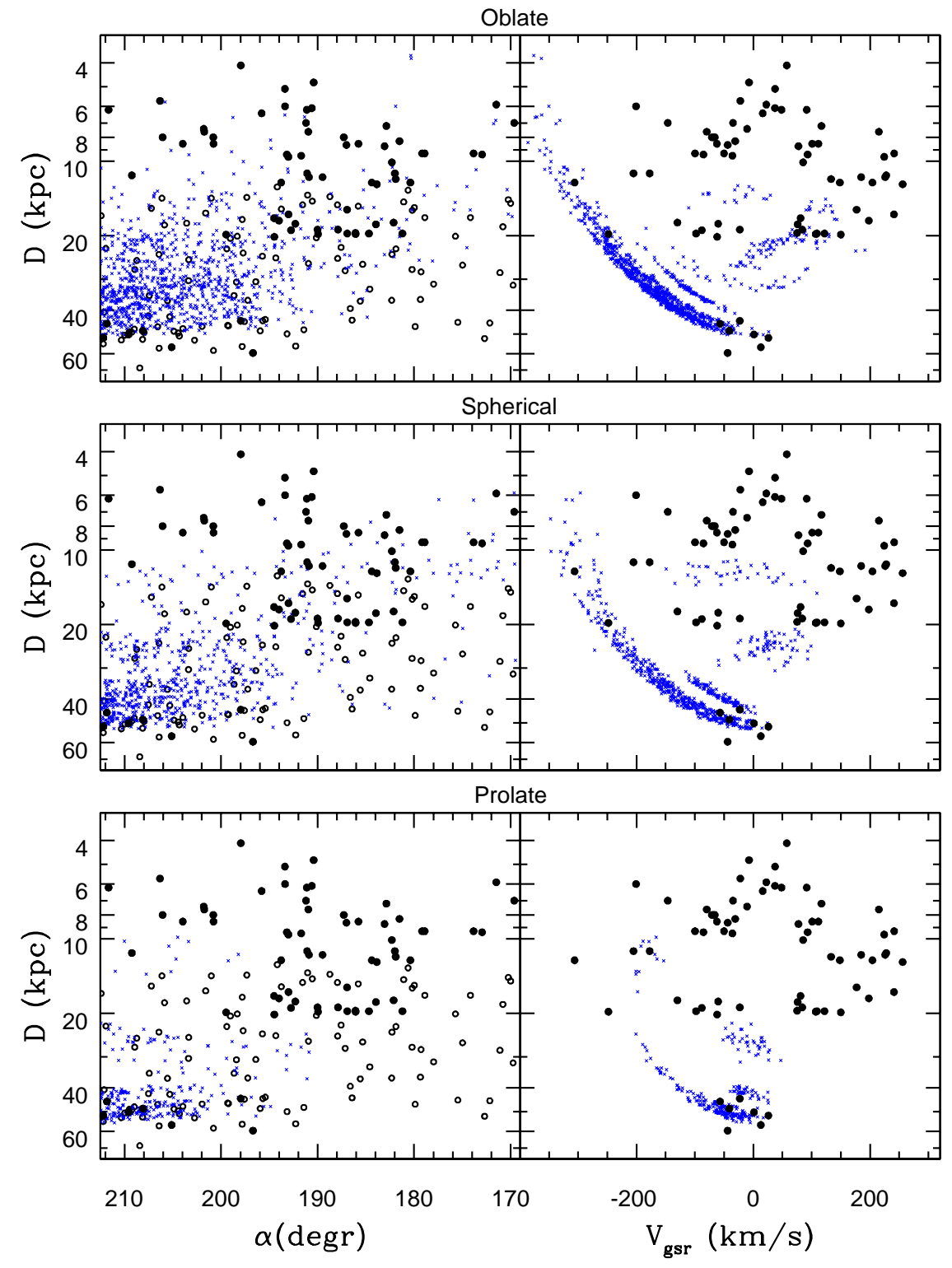

Fig. 9.- Comparison of the location and velocity of the QUEST RRLS with models of the Sgr streams (small crosses) by Law et al. (2005), which assume different shapes for the galactic potential (top: oblate; middle: spherical, bottom: prolate). Filled symbols correspond to different sets of spectroscopic observations of QUEST RRLS: stars with $D<$ $12.5 \mathrm{kpc}$ (Table 3); $16<D<20 \mathrm{kpc}$ (Duffau et al. 2006), D $>40 \mathrm{kpc}$ (Vivas et al. 2005). The open circles in the left panels show the position of QUEST RRLS that have not been observed spectroscopically. 
Table 1. Telescopes and Instruments

\begin{tabular}{lccccccc}
\hline \hline Observatory & Telescope & Instrument & $\begin{array}{c}\text { Grating } \\
\left(\mathrm{mm}^{-1}\right)\end{array}$ & $\begin{array}{c}\text { Spectral Range } \\
(\AA)\end{array}$ & $\begin{array}{c}\text { Resolution } \\
(\AA)\end{array}$ & $\begin{array}{c}\text { \# Spectra/ } \\
\text { \# Stars }\end{array}$ & Dates \\
\hline ESO/La Silla & $1.5 \mathrm{~m}$ & B\&C & 600 & $3300-5500$ & 3.1 & $1 / 1$ & 2001 Jun 17 \\
KPNO & WIYN & Hydra & 600 & $7100-10000$ & 2.9 & $5 / 3$ & 2003 Mar 11 \\
CTIO & SMARTS 1.5m & R-C & 600 & $3532-5300$ & 4.3 & $42 / 28$ & 2003 May 1-9 \\
CTIO & SMARTS 1.5m & R-C & 600 & $3532-5300$ & 4.3 & $24 / 14$ & 2004 Feb-Jun $^{\mathrm{a}}$ \\
CTIO & SMARTS 1.5m & R-C & 600 & $3532-5300$ & 4.3 & $5 / 4$ & $2006 \mathrm{Jun}^{\mathrm{a}}$ \\
CTIO & SMARTS 1.5m & R-C & 600 & $3532-5300$ & 4.3 & $4 / 2$ & $2007{\mathrm{Feb}-A p r^{\mathrm{a}}}^{2}$ \\
KPNO & WIYN & Hydra & 400 & $3500-6200$ & 7.1 & $9 / 5$ & 2007 Feb 20-21 \\
KPNO & WIYN & Hydra & 600 & $7100-10000$ & 2.9 & $3 / 3$ & 2007 Apr 27, May 2 \\
\hline
\end{tabular}

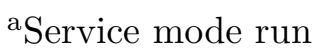


Table 2. Individual Observations of the sample of RRLS ${ }^{\mathrm{a}}$

\begin{tabular}{ccccccrcc}
\hline \hline ID & JD & $\begin{array}{c}\text { Texp } \\
(\mathrm{d})\end{array}$ & Telescope & Phase & $\begin{array}{c}V_{r} \\
\left(\mathrm{~km} \mathrm{~s}^{-1}\right)\end{array}$ & $\begin{array}{c}\sigma_{r} \\
\left(\mathrm{~km} \mathrm{~s}^{-1}\right)\end{array}$ & {$[\mathrm{Fe} / \mathrm{H}]$} \\
\hline 201 & 2452711.7580 & 3600 & WIYN-R & 0.76 & 58 & 5 & $\ldots$ \\
201 & 2452765.6208 & 1200 & SMARTS & 0.02 & 44 & 16 & -1.95 \\
203 & 2452765.6385 & 1200 & SMARTS & 0.02 & 13 & 16 & -1.40 \\
212 & 2452078.4665 & 900 & ESO & 0.56 & 88 & 11 & -2.11 \\
212 & 2452760.6437 & 300 & SMARTS & 0.58 & 109 & 16 & -1.99 \\
\hline
\end{tabular}

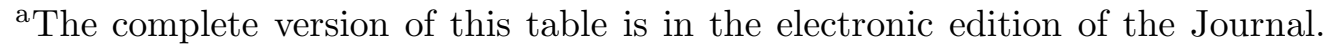
The printed edition contains only a sample.

:Uncertain measurements due to low SNR of the spectrogram. This measurement was not taken into account for calculation of the mean $[\mathrm{Fe} / \mathrm{H}]$ unless it was the only available measurement for the star. 
Table 3. Position, Distance, Velocity and Metallicity of the 43 RRLS $^{\text {a }}$

\begin{tabular}{|c|c|c|c|c|c|c|c|c|c|c|c|c|c|c|}
\hline ID & $\begin{array}{c}\alpha \\
(2000.0)\end{array}$ & $\begin{array}{c}\delta \\
(2000.0)\end{array}$ & $\mathrm{V}_{0}$ & Type & $\begin{array}{l}\text { Period } \\
\text { (d) }\end{array}$ & $\begin{array}{l}\mathrm{HJD}_{0} \\
\text { (d) }\end{array}$ & $\begin{array}{c}r_{\odot} \\
(\mathrm{kpc})\end{array}$ & $\mathrm{N}$ & $\mathrm{N}_{\text {fit }}$ & $\begin{array}{c}V_{\gamma} \\
\left(\mathrm{km} \mathrm{s}^{-1}\right)\end{array}$ & $\begin{array}{c}\sigma_{\text {fit }} \\
\left(\mathrm{km} \mathrm{s}^{-1}\right)\end{array}$ & $\begin{array}{c}\sigma_{\gamma} \\
\left(\mathrm{km} \mathrm{s}^{-1}\right)\end{array}$ & $\begin{array}{c}V_{g s r} \\
\left(\mathrm{~km} \mathrm{~s}^{-1}\right)\end{array}$ & {$[\mathrm{Fe} / \mathrm{H}]$} \\
\hline 151 & 169.625160 & -0.764498 & 14.78 & $\mathrm{c}$ & 0.29370 & 2451611.7002 & 7.0 & 2 & 2 & 90 & 28 & 28 & -35 & -1.32 \\
\hline 157 & 171.487530 & -0.161508 & 14.39 & $a b$ & 0.70840 & 2451571.8154 & 5.9 & 2 & 2 & 142 & 22 & 22 & 22 & -1.67 \\
\hline 160 & 172.957305 & -2.240574 & 15.43 & $a b$ & 0.67619 & 2451582.7058 & 9.4 & 2 & 1 & 217 & $\cdots$ & 20 & 93 & -2.52 \\
\hline 161 & 173.844930 & -0.895030 & 15.40 & $a b$ & 0.60699 & 2451255.6642 & 9.3 & 2 & 2 & 18 & 7 & 15 & -100 & -1.71 \\
\hline
\end{tabular}

${ }^{a}$ The complete version of this table is in the electronic edition of the Journal. The printed edition contains only a sample. 
Table 4. Blue Horizontal Branch from WIYN Observations

\begin{tabular}{cccccc}
\hline \hline ID & $\begin{array}{c}\alpha \\
(\mathrm{deg})\end{array}$ & $\begin{array}{c}\delta \\
(\mathrm{deg})\end{array}$ & $\begin{array}{c}\mathrm{D} \\
(\mathrm{kpc})\end{array}$ & $\begin{array}{c}V_{g s r} \\
\left(\mathrm{~km} \mathrm{~s}^{-1}\right)\end{array}$ & $\begin{array}{c}\sigma_{V_{g s r}} \\
\left(\mathrm{~km} \mathrm{~s}^{-1}\right)\end{array}$ \\
\hline $\mathrm{Q} 245648$ & 190.865875 & -0.926903 & 9.8 & 188 & 8 \\
$\mathrm{Q} 211556$ & 180.395035 & -1.820980 & 9.3 & -6 & 13 \\
$\mathrm{Q} 295219$ & 188.952927 & -2.765785 & 12.0 & 76 & 8 \\
\hline
\end{tabular}

